\title{
Original article (Orijinal araştırma) \\ Mite species of the vegetable crops in Ordu Province with first report of Amblyseius rademacheri Dosse, 1958 (Mesostigmata: Phytoseiidae) in Turkey ${ }^{1}$
}

\author{
Ordu ilinde sebzelerde bulunan akar türleri ile Amblyseius rademacheri Dosse, 1958 \\ (Mesostigmata: Phytoseiidae)'nin Türkiye'de ilk kaydı \\ Mete SOYSAL ${ }^{2}$ \\ Rana AKYAZI ${ }^{\star}$
}

\begin{abstract}
Turkey has suitable ecological conditions to grow a wide variety of vegetables. This research was conducted to investigate mite species on cultivated vegetables that include bean (Phaseolus vulgaris L., Fabaceae), corn (Zea mays L., Poaceae), cucumber (Cucumis sativus L., Cucurbitaceae), eggplant (Solanum melongena L., Solanaceae), leek (Allium porrum L., Alliaceae), lettuce (Lactuca sativa L., Asteraceae), onion (Allium cepa L., Alliaceae), pepper (Capsicum annuum L., Solanaceae), potato (Solanum tuberosum L., Solanaceae), radish (Raphanus sativus L., Brassicaceae), tomato (Solanum lycopersicum L., Solanaceae), watermelon (Citrullus lanatus (Thunb.) Matsum. \& Nakai., Cucurbitaceae) and zucchini (Cucurbita sp., Cucurbitaceae) during 2013-2015 in Ordu Province in the Black Sea Region of Turkey. The samples were taken at weekly intervals from April to October each year. A total of 2030 mite specimens were collected and examined during the study. A total of 43 mite species belonging 15 families and 30 genera were identified. Among them, Tetranychus urticae Koch, 1836 (Prostigmata: Tetranychidae) was found to be the most common phytophagous mite, while Amblyseius swirskii Athias-Henriot, 1962 (Mesostigmata: Phytoseiidae) was the most abundant predator. In addition, Amblyseius rademacheri Dosse, 1958 (Mesostigmata: Pyhtoseiidae) was reported for the first time in Turkey. This new record redescribed and illustrated based on the specimens collected from Solanum melongena L. (Solanaceae). An identification key for the Turkish Amblyseius is also provided.
\end{abstract}

Keywords: Fauna, new record, phytophagous mites, predatory mites, survey, Turkey

\section{Öz}

Türkiye birçok sebze türünün yetişmesi için uygun ekolojik koşullara sahiptir. Bu çalışma 2013-2015 yılları arasında, Ordu ilinde yetiştirilen sebzelerden, fasulye (Phaseolus vulgaris L., Fabaceae), mısır (Zea mays L., Poaceae), hıyar (Cucumis sativus L., Cucurbitaceae), patlıcan (Solanum melongena L., Solanaceae), pirasa (Allium porrum L., Alliaceae), marul (Lactuca sativa L., Asteraceae), soğan (Allium cepa L., Alliaceae), biber (Capsicum annuum L., Solanaceae), patates (Solanum tuberosum L., Solanaceae), turp (Raphanus sativus L., Brassicaceae), domates (Solanum lycopersicum L., Solanaceae), karpuz (Citrullus lanatus (Thunb.) Matsum. \& Nakai., Cucurbitaceae) ve kabak (Cucurbita sp., Cucurbitaceae) üzerinde bulunan akar türlerini belirlemek amacı ile yürütülmüştür. Örneklemeler her yılın nisan-kasım ayları arasında haftalık olarak yapıImıştır. Çalışma boyunca 2030 adet akar toplanmış ve incelenmiştir. Üç alt takıma bağlı 15 familya ve 30 cinsden toplam 43 akar türü saptanmışı̧. Belirlenen akarlar arasında en yaygın bitki zararlısı tür, Tetranychus urticae Koch, 1836 (Prostigmata: Tetranychidae) iken en yaygın predator tür ise Amblyseius swirskii Athias-Henriot, 1962 (Mesostigmata: Phytoseiidae) olmuştur. Ayrıca çalışma da belirlenen Amblyseius rademacheri Dosse, 1958 (Mesostigmata: Pyhtoseiidae) türü, Türkiye predatör akar faunası için yeni kayıt niteliğindedir. Solanum melongena L. (Solanaceae) üzerinden toplanan bu tür tanımlanarak çizimleri sunulmuştur. Ayrıca Türkiye'de tespit edilmiş Amblyseius cinsi akarlar için teşhis anahtarı da verilmiştir.

Anahtar sözcükler: Fauna, yeni kayıt, fitofag akarlar, predator akarlar, survey, Türkiye

\footnotetext{
${ }^{1}$ This manuscript is a part of the master thesis of the first author's and a part of the study was presented as poster in Third International Persian Congress of Acarology (23-25 August 2017, Tehran, Iran) and oral in Central Anatolia Region First Agriculture and Food Congress (International Participated) (26-28 October 2017, Sivas, Turkey) and published as an abstract in the abstracts book.

2 Ordu University, Faculty of Agriculture, Department of Plant Protection, 52200, Ordu, Turkey

* Corresponding author (Sorumlu yazar) e-mail: ranaakyazi@odu.edu.tr

Received (Alınış): 24.07.2018 Accepted (Kabul ediliş): 26.09.2018

Published Online (Çevrimiçi Yayın Tarihi): 07.11.2018
} 
Mite species of the vegetable crops in Ordu Province with first report of Amblyseius rademacheri Dosse, 1958 (Mesostigmata: Phytoseiidae) in Turkey

\section{Introduction}

Due to favorable ecological conditions, many vegetable species are grown in Turkey, with production totaling 30.8 Mt annually Turkey is the forth largest vegetable producer in the world. In Ordu Province, vegetables are produced on about 1300 ha in both open fields and greenhouses. In addition, attempts have been made in Ordu to encourage the production of vegetables especially in greenhouses in recent years. As a result, the total greenhouse vegetable production has increased from $974 \mathrm{t}$ in 2011 to 2,569 t in 2017 (TÜiK, 2017).

Vegetables are indispensible component of daily diet due to their nutritional benefits. They contain many nutrients such as potassium, fiber, folate and vitamins (Abak et al., 2010). However, some mite species such as Tetranychus urticae Koch, 1836 (Prostigmata: Tetranychidae) and Aculops lycopersici (Tryon, 1917) (Trombidiformes: Eriophyidae) are of important pests of vegetable crops causing significant reduction in both yield and quality (Karagöz, 2010). Consequently, there are many predatory mites, especially belonging to family phytoseiidae [e.g. Amblyseius swirskii Athias-Henriot, 1962, Neoseiulus californicus (McGregor, 1954), Phytoseiulus persimilis Athias-Henriot, 1957 (Mesostigmata: Phytoseiidae)], found in association with these phytophagous mites (Al-Atawi, 2011; Kade et al., 2011; Radonjic \& Hrncic, 2011; Binisha \& Bhaskar, 2013; Şekeroğlu \& Kazak, 1993).

A number of studies have been conducted on the mite species on vegetable crops in the different regions of Turkey. Soysal \& Yayla (1988) identified T. urticae and T. cinnabarinus and their predator Phytoseius finitimus Ribaga, 1904 (Mesostigmata: Phytoseiidae) on eggplant in Antalya. In the same region, Çobanoğlu (1989a) determined seven phytoseiid species on vegetables. Polyphagotarsonemus latus (Banks) (Prostigmata: Tarsonemidae) has been reported on peppers in the Mediterranean Region (Yabaş \& Ulubilir, 1995). Five phytophagous and three predatory mite species were determined in Şanlıurfa (Çıkman et al., 1996). Hıncal et al. (2002) identified A. lycopersici and its predator Pronematus ubiquitis (McGregor) (Trombidiformes: Tydeidae) on tomato in İzmir. Phytoseiulus persimilis was determined in many vegetables in southern Turkey (Şekeroğlu \& Kazak, 1993) and cucumber greenhouses of the Samsun Province (Akyazı \& Ecevit, 2008). Kılıç et al. (2012) found that the most common phytophagous species was Rhizoglyphus robini Claparede 1869 (Trombidiformes: Acaridae) and the most common predacious species was Macrocheles merdarius Berlese, 1889 (Trombidiformes: Macrochelidae) in fresh onion fields. Çobanoğlu \& Kumral (2014) identified 34 mite species in tomato growing areas of Ankara, Bursa and Yalova Provinces.

However, so far, no studies have been conducted on the mite species on vegetable crops in Ordu Province. The aim of the study was to investigate the mite species on vegetables crops that are grown both in the open field and in greenhouses in Ordu Provinces between 2013 and 2015.

\section{Material and Methods}

\section{Sampling}

This study was conducted over the growing seasons in Ordu Province between 2013 and 2015. Samples were collected at 10-15 days intervals between April and November each year. Leaf samples were taken from 13 vegetable species, bean (Phaseolus vulgaris L., Fabaceae), corn (Zea mays L., Poaceae), cucumber (Cucumis sativus L., Cucurbitaceae), eggplant (Solanum melongena L., Solanaceae), leek (Allium porrum L., Alliaceae), lettuce (Lactuca sativa L., Asteraceae), onion (Allium cepa L., Alliaceae), pepper (Capsicum annuum L., Solanaceae), potato (Solanum tuberosum L., Solanaceae), radish (Raphanus sativus L., Brassicaceae), tomato (Solanum lycopersicum L., Solanaceae), watermelon (Citrullus lanatus (Thunb.) Matsum. \& Nakai., Cucurbitaceae) and zucchini (Cucurbita sp., Cucurbitaceae). The samples were collected from 209 locations in 106 villages in 13 municipalities of Ordu Province (Figure 1). A total of 863 samples were collected; 794 in open fields and 69 in greenhouses (Table 1). The size of the sample varied according to the size of the sampling area and vegetable species; $25-30$ leaf samples were taken from the large leafy vegetables such as $C$. sativus and Cucurbita sp., 10-20 leaf samples were collected from small leafy vegetables such as $P$. vulgaris and S. Iycopersicum (Anonymous, 2008). Leaf samples were collected randomly from the lower, middle and upper parts of the plants. The samples were placed in paper bags. All bags were then packed in sealed plastic bags. The samples were kept in a refrigerator at $4^{\circ} \mathrm{C}$ (Toros, 1974; Madanlar, 1991). 


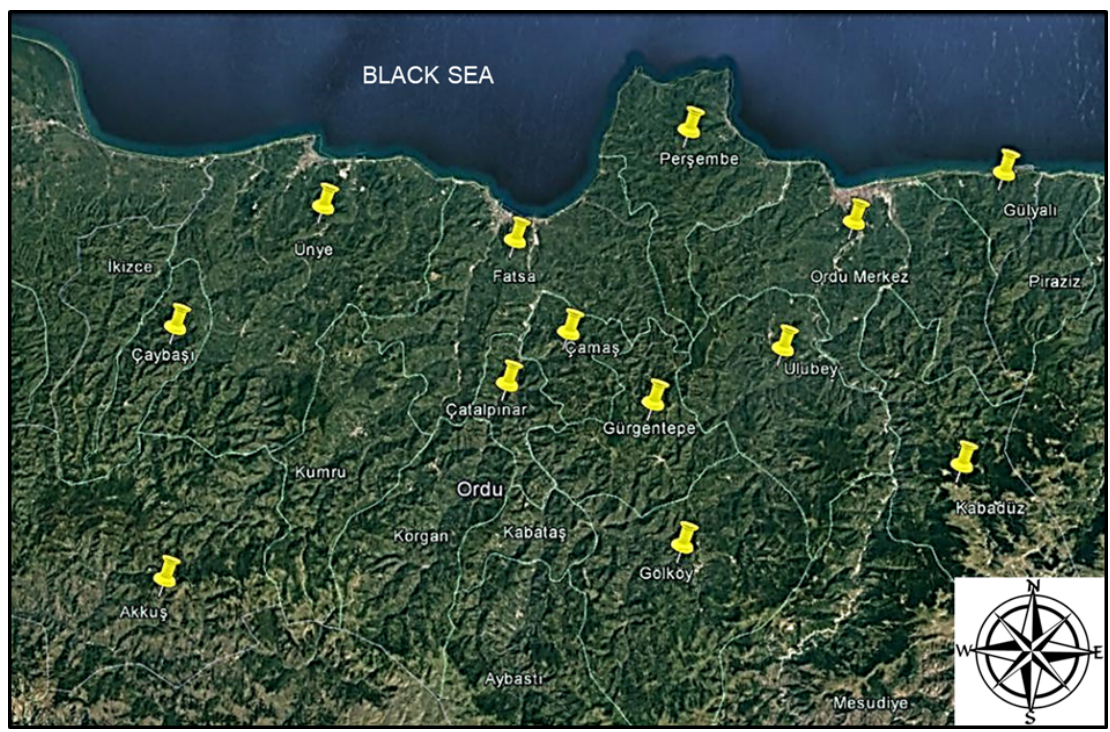

Figure 1. Municipalities sampled in Ordu Province in the Black Sea Region of Turkey (Adapted from www.google.com/maps).

Table 1. List of vegetable species sampled from open fields and greenhouses during growing season in $2013-2015$ in 13 municipalities of Ordu Province, Turkey

\begin{tabular}{llcc}
\hline & Veaetable species sambled & \multicolumn{2}{c}{ Number of sambles } \\
Familv & \multicolumn{1}{c}{ Species } & Open- field & Greenhouse \\
\hline Fabaceae & Phaseolus vulaaris L. & 175 & 10 \\
Solanaceae & Capsicum annuum L. & 150 & 12 \\
Cucurbitaceae & Cucurbita sp. & 99 & 1 \\
Solanaceae & Solanum melonaena L. & 93 & 7 \\
Solanaceae & Solanum Ivcopersicum L. & 90 & 22 \\
Poaceae & Zea mavs L. & 67 & - \\
Cucurbitaceae & Cucumis sativus L. & 62 & 17 \\
Alliaceae & Allium cepa L. & 23 & - \\
Alliaceae & Allium porrum L. & 16 & - \\
Cucurbitaceae & Citrullus lanatus (Thunb.) & 8 & - \\
Asteraceae & Lactuca sativa L. & 5 & - \\
Brassicaceae & Raphanus sativus L. & 3 & - \\
Solanaceae & Solanum tuberosum L. & 3 & 69 \\
\hline Total & & 794 & \\
\hline
\end{tabular}

\section{Extraction, mounting and identification of mite specimens}

Mites found on the adaxial and abaxial surfaces of the leaves were collected under a stereomicroscope (Leica S8 APO, Heerbrugg, Switzerland)) with a fine sable-hair brush. Subsequently, the leaf samples were placed in a Tullgren funnel to extract the mites. All mites collected were preserved in $70 \%$ ethyl alcohol (Ecevit, 1976; Krantz \& Walter, 2009). Mites, except eriophyoids, were cleared in lactophenol solution and mounted in Hoyer's medium (Krantz \& Walter, 2009). The eriophyoid specimens were cleared in Keifer's booster medium and slides were mounted using "F" medium as suggested by Amrine \& Manson (1996). The slides were dried in an oven at $50^{\circ} \mathrm{C}$ for $5-7$ days. 
Identification of mites at the species level was performed using the available taxonomic references such as Zhang (2000, 2003), Ueckermann (2013a) for Tarsonemidae; Pritchard \& Baker (1955), Zhang et al. (2002), Zhang (2003), Seeman \& Beard (2011), Ueckermann \& Çobanoğlu (2012), Auger et al. (2013) for Tetranychidae; Edward \& Donald (1987), Ueckermann \& Çobanoğlu (2012), Çobanoğlu et al. (2016) for Tenuipalpidae; Muma \& Denmark, (1970), Rowell et al. (1978), Çobanoğlu (1989a,b,c, 1993a,b,c,d), Faraji et al. (2007, 2011) for Phytoseiidae; Gonzalez-Rodrigeuz (1965), Fan \& Zhang (2005) for Stigmaeidae; Fain et al. (1999), Yeşilayer \& Çobanoğlu (2012) for Cheyletidae; Skvarla et al. (2014) for Cunaxidae; Solarz (2012) for Acaridae; Ripka et al. (2013), Ueckermann (2013b) for Iolinidae, Tydeidae and Triophtydeidae; Atyeo (1960) for Bdellidae. Mite species were identified under a light microscope (Leica DM 2500) equipped with phase contrast. The mite specimens were deposited in the Mite Collection at the Ordu University, Agricultural Faculty, Plant Protection Department, Ordu, Turkey.

\section{Results and Discussion}

A total of 43 mite species belonging to 15 families were identified (Table 2), with Amblyseius rademacheri Dosse, 1958 (Mesostigmata: Phytoseiidae) as a new record for Turkey. A total of 2030 mites were collected and identified during the study. Tetranychidae ( 1 species, $58 \%$ ) is the most common family followed by Phytoseiidae (15 species 14.2\%), Tarsonemidae (8 species, $11.3 \%$ ), lolinidae (2 species, 4.5\%), Eriophyidae (1 species, 4.4\%), Tydeidae (3 species, 0.9\%), Tenuipalpidae (2 species, $1.2 \%)$, Stigmaeidae (1 species, $0.4 \%$ ), Eupodidae (1 species, $0.3 \%)$, Acaridae (2 species, $0.2 \%)$, Erythraeidae (1 species, $0.1 \%$ ), Trombidiidae (1 species, $0.1 \%)$, Triophtydeidae (2 species, $0.1 \%$ ), Cheyletidae (2 species, $0.1 \%$ ) and Bdellidae (1 species, $0.05 \%$ ). The most common phytophagous species were $T$. urticae (58\%), P. latus (9.4\%) and A. lycopersici (4.4\%). Among the predators, the most common species was Homeopronematus staerki (Schruft, 1972) (Trombidiformes: Iolinidae) (4.2\%) followed by $A$. swirskii (3.6\%) and P. persimilis (3.1\%).

Table 2. Distribution of mite species on vegetable crops from the greenhouses and open fields in the Ordu Province between 2013 and 2015

\begin{tabular}{|c|c|c|c|c|c|c|c|c|c|c|c|c|c|c|c|c|c|}
\hline Family & Species & Open field & Greenhouse & $\%$ & $\mathrm{Pv}$ & $\mathrm{Ca}$ & $\mathrm{Cu}$ & Sm & $\mathrm{SI}$ & $\mathrm{Zm}$ & Cs & Ac & $\mathrm{Ap}$ & $\mathrm{Cl}$ & Ls & Rs & St \\
\hline & Amblyseius andersoni & 6 & 1 & 0.3 & & + & & * & & & + & & & & & & \\
\hline & Amblyseius rademacheri & 2 & - & 0.1 & & & & + & & & & & & & & & \\
\hline & Amblyseius swirskii & 73 & - & 3.6 & + & + & + & + & & & + & & & & & & \\
\hline & Aristadromips masseei & 9 & 6 & 0.7 & & & + & & & & $+^{*}$ & & & & & & \\
\hline & Euseius finlandicus & 7 & - & 0.3 & + & + & & & & & + & & & & & & \\
\hline & Euseius gallicus & 20 & - & 1.0 & + & + & & & & & + & & & & & & \\
\hline & Kampimodromus aberrans & 2 & - & 0.1 & & & + & & & & + & & & & & & \\
\hline \multirow[t]{8}{*}{ Phytoseiidae } & Neoseiulus barkeri & 7 & 1 & 0.4 & + & & + & + & + & & * & & & & & & \\
\hline & Neoseiulus bicaudus & 1 & - & 0.0 & & & & & & & & + & & & & & \\
\hline & Neoseiulus californicus & 2 & 15 & 0.8 & & * & & + & & + & * & & & & & & \\
\hline & Phytoseius finitimus & 27 & 2 & 1.4 & + & + & + & + & + & & $+^{*}$ & & & & & & \\
\hline & Phytoseiulus persimilis & 46 & 17 & 3.1 & + & * & + & + & + & & $+^{*}$ & & & & & & \\
\hline & Proprioseiopsis okanagensis & 1 & - & 0.0 & & & + & & & & & & & & & & \\
\hline & Transeius wainsteini & 5 & - & 0.2 & & + & + & & & & + & & & & & & \\
\hline & Typhlodromus athiasae & - & 38 & 1.9 & & * & & & & & * & & & & & & \\
\hline
\end{tabular}


Table 2. (Continued)

\begin{tabular}{|c|c|c|c|c|c|c|c|c|c|c|c|c|c|c|c|c|c|}
\hline \multirow{2}{*}{$\frac{\text { Family }}{\text { Bdellidae }}$} & \multirow{2}{*}{$\begin{array}{l}\text { Species } \\
\text { Cyta sp. }\end{array}$} & \multicolumn{2}{|c|}{ Open fieldGreenhouse } & \multirow{2}{*}{$\begin{array}{c}\% \\
0.0\end{array}$} & \multirow[t]{2}{*}{$\mathrm{Pv}$} & \multirow[t]{2}{*}{$\mathrm{Ca}$} & \multirow[t]{2}{*}{$\mathrm{Cu}$} & \multirow{2}{*}{$\frac{\mathrm{Sm}}{*}$} & \multirow[t]{2}{*}{$\mathrm{SI}$} & \multirow[t]{2}{*}{$\mathrm{Zm}$} & \multirow[t]{2}{*}{ Cs } & \multirow[t]{2}{*}{ Ac } & \multirow[t]{2}{*}{$\mathrm{Ap}$} & \multirow[t]{2}{*}{$\mathrm{Cl}$} & \multirow[t]{2}{*}{ Ls } & \multirow[t]{2}{*}{ Rs } & \multirow[t]{2}{*}{ St } \\
\hline & & 1 & - & & & & & & & & & & & & & & \\
\hline \multirow{2}{*}{ Cheyletidae } & Cheletomimus berlesei & 1 & - & 0.0 & & & & + & & & & & & & & & \\
\hline & Cheletogenes ornatus & 1 & - & 0.0 & & & & + & & & & & & & & & \\
\hline Eriophyidae & Aculops Iycopersici & 80 & 9 & 4.4 & & & & & $+^{*}$ & & & & & & & & \\
\hline Erythraeidae & Abrolophus sp. & 3 & - & 0.1 & & & & & & & $+^{*}$ & & & + & & & \\
\hline Eupodidae & Eupodes sp. & 6 & - & 0.3 & & & + & & & & & & & & & & \\
\hline \multirow{2}{*}{ lolinidae } & Pronematus sextoni & 7 & - & 0.3 & + & + & & + & & & + & & & & & & \\
\hline & Homeopronematus staerki & 78 & 7 & 4.2 & $+^{*}$ & $+^{*}$ & + & $+^{*}$ & $+*$ & & + & & & & & & \\
\hline Stigmaeidae & Zetzellia mali & 8 & - & 0.4 & + & & + & + & + & & & & & & & & \\
\hline \multirow{8}{*}{ Tarsonemidae } & Polyphagotarsonemus latus & 187 & 4 & 9.4 & $+^{*}$ & + & + & + & + & + & + & & & & & & \\
\hline & Tarsonemus confusus & 16 & 2 & 0.9 & & & & * & $+^{*}$ & & & & & & & & \\
\hline & Tarsonemus waitei & 10 & 3 & 0.6 & & & + & & * & & + & + & & & & & \\
\hline & Daidalotarsonemus sp. & 1 & - & 0.0 & & & + & & & & & & & & & & \\
\hline & Tarsonemus sp. 1 & 2 & - & 0.1 & & & & & + & & & & & & & & \\
\hline & Tarsonemus sp. 2 & 1 & - & 0.0 & & & & + & & & & & & & & & \\
\hline & Tarsonemus sp. 3 & 1 & - & 0.0 & & & + & & & & & & & & & & \\
\hline & Xenotarsonemus sp. & 2 & - & 0.1 & & + & & & & & & & & & & & \\
\hline \multirow{2}{*}{ Tetranychidae } & Tetranychus urticae (GF) & 938 & 239 & 58. & $+^{*}$ & $+^{*}$ & + & $+^{*}$ & $+^{*}$ & + & $+^{*}$ & + & + & & + & + & \\
\hline & Tetranychus urticae (RF) & 69 & 12 & 4.0 & $+^{*}$ & & & $+^{*}$ & * & + & + & & & & & & \\
\hline \multirow{2}{*}{ Tenuipalpidae } & Brevipalpus lewisi & 12 & - & 0.6 & + & + & & + & & & & & & & & & \\
\hline & Brevipalpus obovatus & 12 & - & 0.6 & + & & & + & + & + & + & & & & & & \\
\hline \multirow{2}{*}{ Triophtydeidae } & Triophtydeus immanis & 2 & - & 0.1 & & & & & + & & & & & & & & \\
\hline & Triophtydeus triophthalmus & 1 & - & 0.0 & + & & & & & & & & & & & & \\
\hline Trombidiidae & Allothrombium pulvinum & 3 & - & 0.1 & + & + & & + & & & & & & & & & \\
\hline \multirow{3}{*}{ Tydeidae } & Brachytydeus mali & 2 & - & 0.1 & + & + & & & & & & & & & & & \\
\hline & Tydeus californicus & 2 & - & 0.1 & & + & & & & & & & & & & & \\
\hline & Tydeus goetzi & 15 & - & 0.7 & + & + & + & + & & & + & & & & & & \\
\hline \multirow{3}{*}{ Acaridae } & Tyrophagus palmarum & 2 & - & 0.1 & & & & & & + & + & & & & & & \\
\hline & Tyrophagus putrescentiae & 3 & - & 0.1 & + & & & + & & & & & & & & & \\
\hline & TOTAL & 1674 & 356 & & 18 & 19 & 17 & 22 & 13 & 5 & 21 & 3 & 1 & 1 & 1 & 1 & - \\
\hline
\end{tabular}

\%: percentage in total mites; GF: green form; RF: red form; +: open field, *: greenhouse; Pv: Phaseolus vulgaris, Ca: Capsicum annuum, Sm: Solanum melongena, Cu: Cucurbita sp., SI: Solanum lycopersicum, Zm: Zea mays, Cs: Cucumis sativus, Ap: Allium porrum, Ac: Allium cepa, Ls: Lactuca sativa, Cl: Citrullus lanatus, Rs: Raphanus sativus, St: Solanum tuberosum

\section{Tetranychidae}

\section{Tetranychus urticae Koch, 1836}

Open field green form:

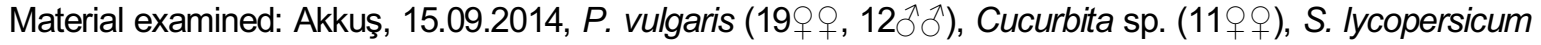
(2우); Çamaş, 21.08.2014, Z. mays (1ㅇ), 05.08.2014, P. vulgaris (8우), S. melongena (3우), C. sativus (2우), 
Z. mays (4우); Çatalpınar, 05.08.2014, P. vulgaris (5우), 21.08.2014, P. vulgaris (24우, $\left.15{ }^{\lambda}{ }^{\lambda}\right)$, Cucurbita sp.

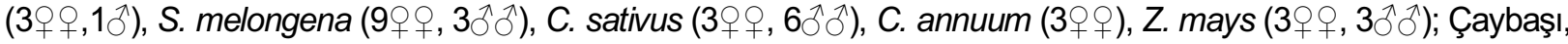

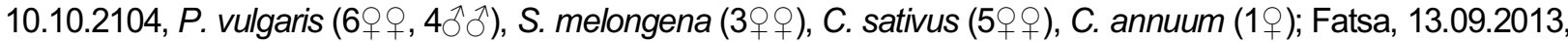

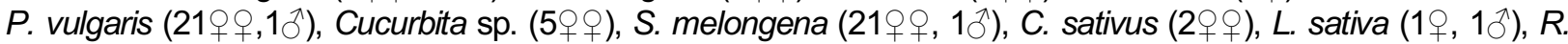

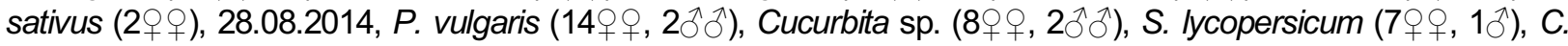

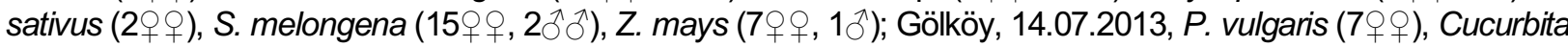

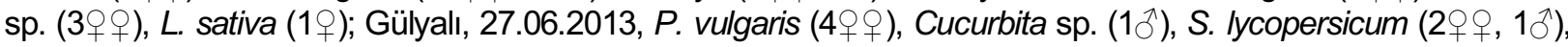

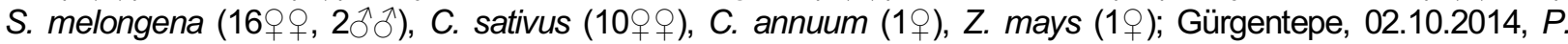
vulgaris $(43 ㅇ ㅜ, 22 \hat{\jmath})$, Cucurbita sp. $(15 ㅇ ㅜ, 1 \hat{\jmath})$, S. melongena $(11$ 웅, $1 \hat{\jmath})$, C. sativus $(3 ㅇ ㅜ, 1 \hat{\jmath})$, C. annuum

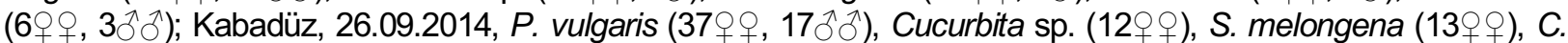

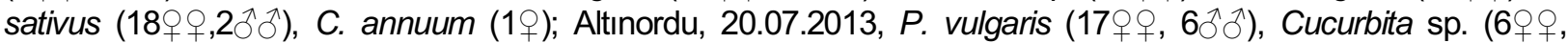

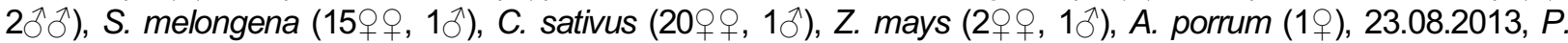
vulgaris (20우), Cucurbita sp. (8우), S. melongena (7우), C. sativus (1ㅇ), C. annuum (1ㅇ), Z. mays (1우); Perşembe, 23.07.2013, C. sativus (13우), 27.09.2013, P. vulgaris (8우), Cucurbita sp. (18우, $\left.1{ }^{\Uparrow}\right), S$.

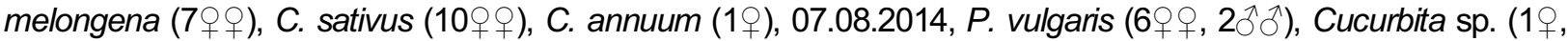

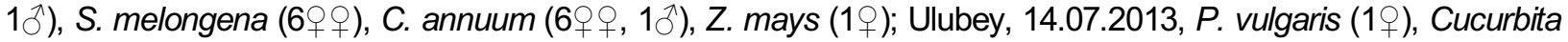

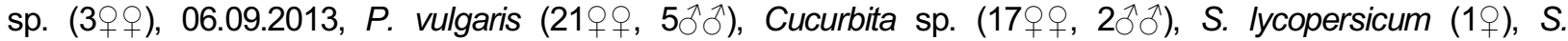
melongena (11우, $7 \hat{\jmath})$ ), C. sativus (21웅), C. annuum (5웅), Z. mays (1우), 11.09.2014, P. vulgaris (15우우,

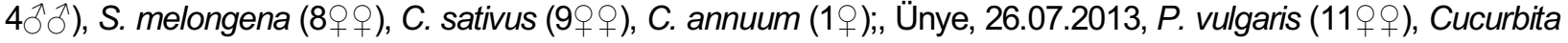
sp. (8우), S. lycopersicum (2우), S. melongena (2qㅇ), C. sativus (1ㅇ), C. annuum (1ㅇ), A. porrum (1우),

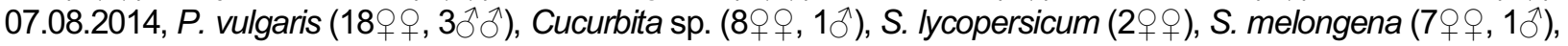
C. sativus (1ㅇ), Z. mays (4우), A. cepa (1ㅇ), A. porrum $(2+$ 우, $1 \hat{)})$.

Open field red form:

Material examined: Akkuş, 15.09.2014, P. vulgaris (1 $\left.{ }^{\lambda}\right)$; Çamaş, 05.08.2014, P. vulgaris $\left(5 \circ\right.$ 우, $\left.1 \delta^{\lambda}\right), C$.

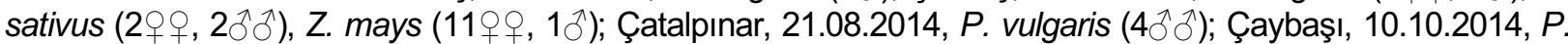

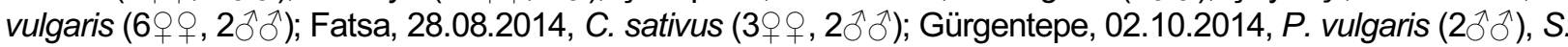

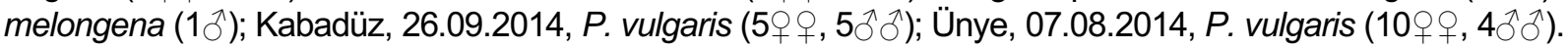

Greenhouse green form:

Material examined: Çaybaşı, 10.10.2014, C. sativus (3우), C. annuum (19); Fatsa, 13.09.2013, $P$.

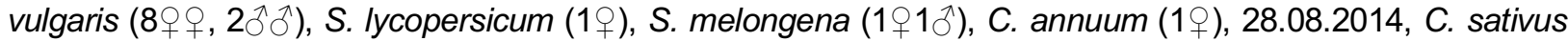
(2우, $2 \hat{\jmath}$ ); Gülyalı, 27.06.2013, P. vulgaris (23웅), C. sativus (15웅) , S. lycopersicum (1우); Gürgentepe,

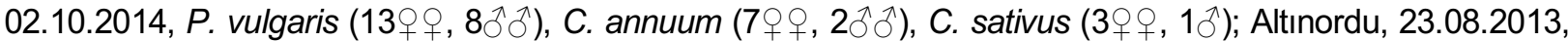

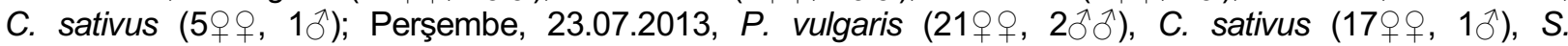

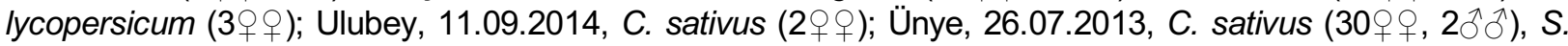
melongena (33우, 1 $\delta^{\lambda}$ ), S. lycopersicum (13우), 07.08.2014, C. sativus (10우), S. lycopersicum (3웅).

Greenhouse red form:

Material examined: Gürgentepe, 02.10.2014, P. vulgaris (1+); Ünye, 07.08.2014, S. lycopersicum

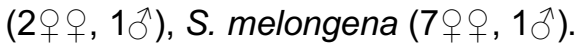

Distribution: T. urticae is a cosmopolitan polyphagous mite species (Migeon et al., 2011). In previous studies, it was commonly found on crops in Turkey (Öngören et al., 1975; Soysal \& Yayla, 1988; Güven \& Madanlar, 2000; Tokkamış \& Yanar, 2011; Çobanoğlu \& Kumral, 2014, 2016; Akyazı et al., 2017; İnak \& Çobanoğlu, 2018).

\section{Tenuipalpidae}

\section{Brevipalpus obovatus Donnadieu, 1875}

Material examined (open field): Çamaş, 05.08.2014, P. vulgaris (19); Gülyall, 19.08.2015, S. lycopersicum (1pn), C. sativus (1ㅇ); Kabadüz, 26.08.2014, S. melongena (1)); Altınordu, 29.08.2013, S. melongena (1ㅇ, 3pn); Perşembe, 27.09.2013, S. melongena (2qo+), Z. mays (1ㅇ); Ulubey, 06.09.2013, C. sativus (1pn). 
Distribution: B. obovatus has been found on many types of plants worldwide (Beard et al., 2012). In Turkey, this species was determined for the first time on lemon trees (Düzgüneş, 1952), and subsequently in various localities in Turkey (Çobanoğlu et al., 2016).

\section{Brevipalpus lewisi McGregor, 1949}

Material examined (open field): Fatsa, 28.08.2014, S. melongena (1+); Perşembe, 27.09.2013, $P$. vulgaris (1, $1 \mathrm{pn}), S$. melongena (1ㅇ), C. annuum (5우).

Distribution: B. lewisi occurs on many plant groups around the world and Turkey (Jeppson et al., 1975; Soylu \& Ürel, 1977; Ghai \& Shenhmar, 1984; Göven et al., 2009; Khanjani et al., 2013; Yanar \& Erdoğan, 2013; Çobanoğlu et al., 2016; Ueckermann \& Ripka, 2016; Ueckermann et al., 2018).

\section{Tarsonemidae}

\section{Polyphagotarsonemus latus (Banks)}

Material examined (open field): Çamaş, 05.08.2014, P. vulgaris (4우), C. sativus (2q⿱㇒, $1 \hat{\jmath})$;

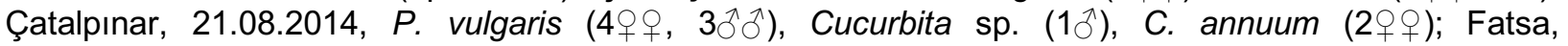
13.09.2013, P. vulgaris (9우), C. annuum (2qㅇ)); Gürgentepe, 02.10.2014, P. vulgaris (1ㅇ); Kabadüz, 26.09.2014, P. vulgaris (1ㅇ), Cucurbita sp. (8우, $\left.1{ }^{\Uparrow}\right)$, S. melongena (14우), C. annuum (1ㅇ); Altınordu,

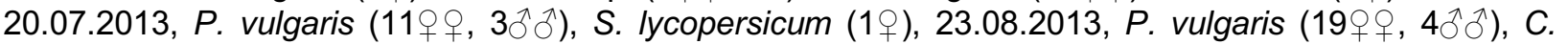

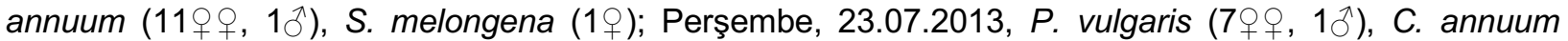

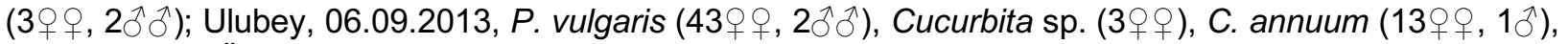

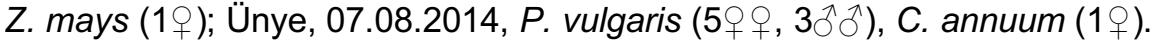

Material examined (greenhouse): Fatsa, 13.09.2013, P. vulgaris (4 $q$ ).

Distribution: P. latus is a very common species around the world (Binisha \& Bhaskar 2013; CABI, 2014). In Turkey, it has been recorded in association with several vegetable crops (Çobanoğlu, 1995; Tunç \& Göçmen, 1995; Yabaş \& Ulubilir, 1995; Bulut, 1999; Can \& Çobanoğlu, 2010).

\section{Daidalotarsonemus sp. De Leon, 1956}

Material examined (open field): Çatalpınar, 21.08.2014, Cucurbita sp. (1ㅇ).

\section{Tarsonemus waitei Banks, 1912}

Material examined (open field): Fatsa, 13.09.2013, Cucurbita sp. (7우), C. sativus (2qo+); Ünye, 26.07.2013, A. cepa (1).

Material examined (greenhouse): Fatsa, 13.09.2013, S. lycopersicum (19); Ulubey, 06.09.2013, S. lycopersicum (2우).

Distribution: T. waitei previously recorded in Brazil, Canada, China, Congo, Costa Rica, Hungary, New Zealand, Poland, Portugal and Ukraine (Lin \& Zhang, 2002; Ripka et al., 2005). It has also been found in Turkey (Çobanoğlu, 1995; Tokkamış, 2011).

\section{Tarsonemus confusus Ewing, 1939}

Material examined (open field): Gürgentepe, 20.10.2014, S. lycopersicum (15우, 1ठ).

Material examined (greenhouse): Fatsa, 28.08.2014, S. melongena (1+1); Ulubey, 06.09.2013, S. lycopersicum (1ㅇ).

Distribution: T. confusus was reported in the USA, Canada, Italy, Ireland, Germany, Poland, Ukraine, Russia, Japan, Korea, China, Egypt by Lin \& Zhang (2002) and Hungary by Ripka et al. (2005). In Turkey, it was first identified on Pyracantha coccinea Roem (Rosaceae) in Edirne by Çobanoğlu (1995). C. annuum, C. sativus (Tokkamış, 2011) and S. lycopersicum (Çobanoğlu \& Kumral, 2014) are among the hosts of T. confusus. 


\section{Xenotarsonemus sp.}

Material examined (open field): Gülyalı, 10.08.2015, C. annuum (2우).

\section{Tarsonemus sp. 1}

Material examined (open field): Gürgentepe, 20.10.2014, S. lycopersicum (1); Kabadüz, 26.09.2014, S. lycopersicum (19).

\section{Tarsonemus sp. 2}

Material examined (open field): Gülyalı, 10.07.2013, S. melongena (19).

\section{Tarsonemus sp. 3}

Material examined (open field): Fatsa, 28.08.2014, Cucurbita sp. (1ㅇ).

\section{Eriophyidae}

\section{Aculops lycopersici (Tryon, 1917)}

Material examined (open field): Çatalpınar, 05.08.2014, S. lycopersicum (10우); Fatsa, 13.09.2013, S. lycopersicum (9q⿱㇒); Kabadüz, 26.09.2014, S. lycopersicum (4우); Altınordu, 20.07.2013, S. lycopersicum (26우), 23.08.2013, S. lycopersicum (3ㅇ); Perşembe, 27.09.2013, S. lycopersicum (13우); Ulubey, 06.09.2013, S. lycopersicum (7우); Ünye, 07.08.2014, S. lycopersicum (8웅).

Material examined (greenhouse): Fatsa, 13.09.2013, S. Iycopersicum (9웅).

Distribution: A. lycopersici is widespread worldwide (Denizhan et al., 2015). It has also been recorded in association with tomato plants in many localities in Turkey (Şekeroğlu \& Özgür, 1984; Madanlar \& Öncüer, 1994; İnal, 2005; Yanar et al., 2008; Can \& Çobanoğlu, 2010; Çobanoğlu \& Kumral, 2014; Denizhan et al., 2015).

\section{Tydeidae}

\section{Brachytydeus mali (Oudemans, 1929)}

Material examined (open field): Çatalpınar, 21.08.2014, P. vulgaris (19); Gülyalı, 19.08.2015, C. annuum (19).

Distribution: B. mali was collected in Scotland (Baker \& Wharton, 1952), Serbia (Stojnic et al., 2002), Iran (Jalilirad et al., 2012), Spain and Greece (Anonymous, 2015). It was reported in Turkey (Istanbul) by Yeşilayer (2009).

\section{Tydeus californicus (Banks, 1904)}

Material examined (open field): Akkuş, 15.09.2014, C. annuum (1)); Perşembe, 13.09.2013, C. annuum (19).

Distribution: T. californicus is very common around the world (Tempfli et al., 2015). The first record of $T$. californicus in Turkey was on citrus leaves in Adana (Düzgüneş, 1963).

\section{Tydeus goetzi Schruft, 1972}

Material examined (open field): Akkuş, 15.09.2014, C. annuum (19); Gülyalı, 27.06.2013, C. sativus (1), 10.07.2013, P. vulgaris (2우), C. annuum (2o+q); Perşembe, 27.09.2013, P. vulgaris (1ㅇ), C. annuum (2우), 09.07.2014, Cucurbita sp. (1ㅇ); Altınordu, 20.07.2013, P. vulgaris (2우), C. sativus (2우), S. melongena (1ㅇ).

Distribution: T. goetzi is a rare species. This mite has been determined only in Germany (Schruft, 1972), France (Andre, 2011) and Turkey (Akyazı et al., 2017). 


\section{Triophtydeidae}

\section{Triophtydeus triophthalmus (Oudemans, 1929)}

Material examined (open field): Ulubey, 11.09.2014, P. vulgaris (19).

Distribution: T. triophthalmus has been reported from Italy (Sabbatini Peverieri et al., 2009), Ukraine (Ripka et al., 2005), Turkey (Özman-Sullivan et al., 2005), Germany and Sweden (Tempfli et al., 2015). However, the feeding habits of $T$. triophthalmus is contradictory (Tempfli et al., 2015).

\section{Triophtydeus immanis Kuznetzov, 1973}

Material examined (open field): Altınordu, 21.07.2013, S. lycopersicum (1), 23.08.2013, S. lycopersicum (1ㅇ).

Distribution: T. immanis has been reported from South Africa (Ueckermann \& Grout, 2007), Turkey (Özman-Sullivan et al., 2005) and Hungary (Ripka et al., 2002, 2005).

\section{Iolinidae}

\section{Homeopronematus staerki (Schruft, 1972)}

Material examined (open field): Akkuş, 15.09.2014, P. vulgaris (1), C. annuum (7우); Çamaş, 05.08.2014, P. vulgaris (2o+ ), Cucurbita sp. (1+); Çatalpınar, 21.08.2014, S. lycopersicum (2o $q$ ); Fatsa, 13.09.2013, P. vulgaris (2우), Cucurbita sp. (3우), C. annuum (1)); Gülyalı, 10.07.2013, P. vulgaris (2ㅇ); Gürgentepe, 02.10.2014, P. vulgaris (6q+); Kabadüz, 26.09.2014, P. vulgaris (3q⿱, 1tn), $S$. lycopersicum (7우), S. melongena (2우); Altınordu, 20.07.2013, P. vulgaris (3우), S. lycopersicum (5우), C. sativus (1ㅇ), 23.08.2013, P. vulgaris (5우), S. lycopersicum (1ㅇ); Perşembe, 23.07.2013, S. lycopersicum (2qo), 27.09.2013, S. Iycopersicum (4ㅇ), C. annuum (2o+ + ), 09.07.2014, P. vulgaris (1ㅇ); Ulubey, 14.07.2013, P. vulgaris (2우), 06.09.2014, P. vulgaris (5우), C. sativus (1ㅇ), $S$. melongena (1); Ünye, 26.07.2013, $P$. vulgaris (3우), 07.08.2014, $P$. vulgaris (2우).

Material examined (greenhouse): Perşembe, 23.07.2013, P. vulgaris (1ㅇ), S. lycopersicum (3우); Ünye, 26.07.2013, C. annuum (2우); Fatsa, 13.09.2013, S. melongena (1ㅇ).

Distribution: H. staerki has been reported in Serbia (Stojnic et al., 2002), Germany (Schruft, 2006), Hungary (Ripka et al., 2005, 2013; Tempfli et al., 2015). Özman-Sullivan et al. (2005) recorded $H$. staerki on hazelnut in Turkey.

\section{Pronematus sextoni Baker, 1968}

Material examined (open field): Fatsa, 13.09.2013, P. vulgaris (3ㅇ+t); Altınordu, 23.08.2013, S. melongena (1), C. annuum (1); Ulubey, 06.09.2013, C. sativus (1tn), C. annuum (1).

Distribution: P. sextoni has been found in India (Gupta, 1985), Africa (Gupta et al., 2015) and Turkey (Çobanoğlu \& Kazmierski, 1999).

\section{Cheyletidae}

\section{Cheletomimus berlesei (Oudemans)}

Material examined (open field): Altınordu, 23.08.2013, S. melongena (1 + ).

Distribution: $C$. berlesei has been reported from America, France, Italy, Israel and New Zealand (Summers \& Price, 1970). It Turkey, it was first identified in Istanbul by Yeşilayer \& Çobanoğlu (2012) and it was found in association with Cenopalpus lineola Canestrini \& Fanzago (an ornamental plant). During this study, it was collected with $T$. urticae, $P$. latus and $P$. sextoni on $S$. melongena. 


\section{Cheletogenes ornatus (Canesterini \& Fanzago, 1876)}

Material examined (open field): Perşembe, 09.07.2014, S. melongena (1+).

Distribution: This mite is found in Southern Europe, South Africa, Italy, Israel, China, Australia and America (Volgin, 1989). C. ornatus was reported by Düzgüneş (1963) on lemon trees in Antalya, Turkey. In this study, it was found together with $T$. urticae on S. melongena.

\section{Stigmaeidae}

\section{Zetzellia mali (Ewing)}

Material examined (open field): Akkuş, 15.09.2014, P. vulgaris (1+ ); Gürgentepe, 02.10.2014, S. melongena (1+); Altınordu, 20.07.2013, S. lycopersicum (2q+), 21.07.2015, Cucurbita sp. (1); Perşembe, 27.09.2013, S. melongena (2우); Ünye, 07.08.2014, P. vulgaris (1ㅇ).

Distribution: Z. mali has an extensive worldwid range (Gerson et al., 2003). It has been widely found in many provinces in Turkey by many researchers (Düzgüneş, 1963; Akyazı \& Ecevit, 2003; Çobanoğlu et al., 2003; İnal, 2005; Kumral, 2005; Kasap \& Çobanoğlu, 2007; Denizhan \& Çobanoğlu, 2009; Yeşilayer, 2009; Karagöz, 2010; Sağlam \& Çobanoğlu, 2010; Dönel \& Doğan, 2013; Çobanoğlu \& Kumral, 2014; Kumral \& Çobanoğlu, 2015b; Akyazı et al., 2016a, 2017; İnak \& Çobanoğlu, 2018). During this study, it was collected together with $T$. urticae, H. staerki and A. lycopersici on P. vulgaris.

\section{Eupodidae}

\section{Eupodes sp.}

Material examined (open field): Ulubey, 11.09.2014, Cucurbita sp. (3우); Gürgentepe, 02.10.2014, Cucurbita sp. (2우); Gülyalı, 27.06.2013, Cucurbita sp. (1ㅇ).

\section{Bdellidae}

\section{Cyta sp.}

Material examined (greenhouse): Fatsa, 28.08.2014, S. melongena (19).

\section{Trombidiidae}

\section{Allothrombium pulvinum Ewing, 1917}

Material examined (open field): Kabadüz, 26.07.2013, S. melongena, (1dn), 26.09.2014, $P$. vulgaris (1亏), C. annuum (1ふ).

Distribution: A. pulvinum was first discovered in North America by Ewing (1917). Later, it was determined in North America, Europe and Asia (Zhang \& Norbakhsh, 1995). It has also been reported in Turkey (Çobanoğlu et al., 2003; Yeşilayer, 2009).

\section{Erythraeidae}

\section{Abrolophus sp.}

Material examined (open field): Ünye, 07.08.2014, C. sativus (1ㅇ), C. lanatus (1ㅇ).

Material examined (greenhouse): Fatsa, 13.09.2013, C. sativus (1).

Distribution: In Turkey, Abrolophus sp. was identified in Bursa (Kumral, 2005), İzmir (Kılıç et al., 2012) and Ankara (Kumral \& Çobanoğlu, 2015a). 


\section{Acaridae}

\section{Tyrophagus putrescentiae (Schrank, 1781)}

Material examined (open field): Çatalpınar, 21.08.2014, P. vulgaris (19); Altınordu, 20.07.2013, S. melongena (1tn); Perşembe, 27.09.2013, P. vulgaris (1へ).

Distribution: The cosmopolitan T. putrescentiae has been found in New Zealand, Australia, China, Ecuador, Germany, Japan, the Netherlands and the USA (Fan \& Zhang, 2007). In Turkey, this species was collected from vegetables including $C$. annuum, C. sativus (Tokkamış \& Yanar, 2011), A. cepa (Kılıç et al., 2012), S. lycopersicum (Çobanoğlu \& Kumral, 2014).

\section{Tyrophagus palmarum (Oudemans, 1924)}

Material examined (open field): Çatalpınar, 21.08.2014, Z. mays (19), Ulubey, 06.09.2013, C. sativus (19).

Distribution: T. palmarum has been reported from Czechoslovakia (Zdarkova, 1967), Germany (Franz et al., 1997), Australia, Netherland, Tuvalu and New Zealand (Fan \& Zhang, 2007). It was detected for the first time in Turkey in dust samples taken from the homes of allergic asthmatic patients in Samsun Province of Turkey by Çelik (2009).

\section{Phytoseiidae}

Key to species of the genus Amblyseius Berlese in Turkey based on adult females (based on Faraji et al., 2011; Akyazı et al., 2016b)

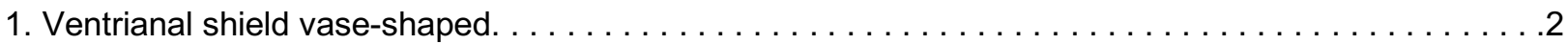

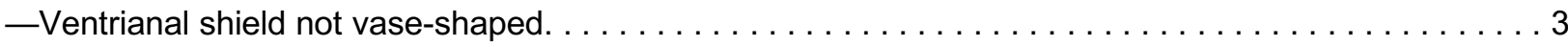

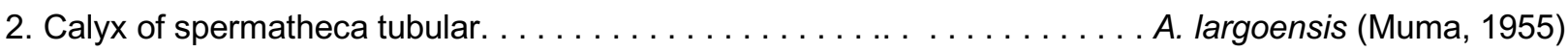

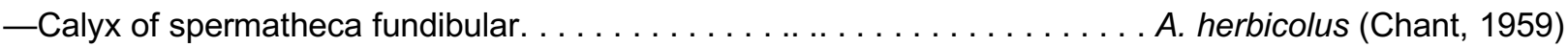

3. Seta Z5 longer than width of dorsal shield; spermatheca with calyx annulated, flared distally...........

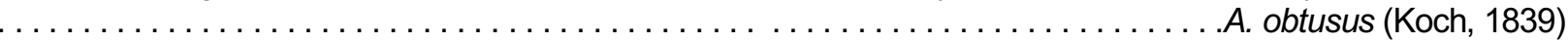

—Seta Z5 shorter than width of dorsal shield; spermatheca with calyx not annulated. . . . . . . . .4

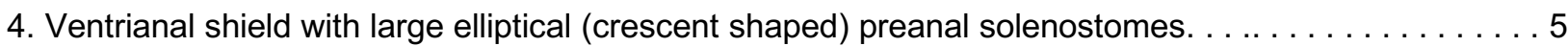

-Ventrianal shield with small round preanal solenostomes. $\ldots \ldots \ldots \ldots \ldots \ldots \ldots \ldots \ldots$

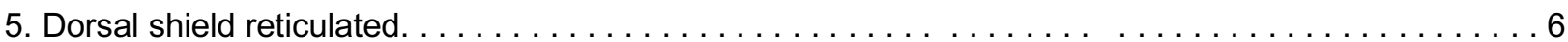

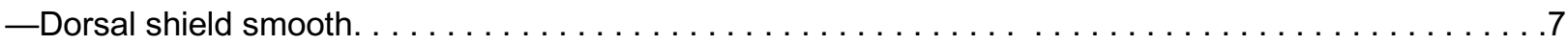

6. Fixed digit with 10 teeth; Gell with 8 setae . . . . . . . . . . . . . . A. bryophilus Karg, 1970

—Fixed digit with 7-8 teeth; Gell with 7 setae. . . . . . . . . . . . . . . . . . . rademacheri Dosse, 1958

7. Seta Z5 102-116 long; atrium of spermatheca relatively long; StilV at most reaching the insertion of

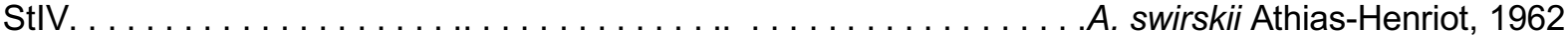

- Seta Z5 longer than 150; atrium of spermatheca short and c-shaped; StilV passing well behind the

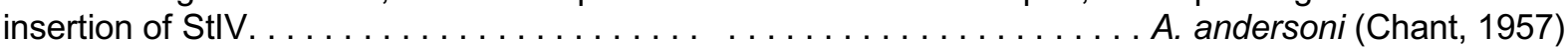

8. Seta Z4 almost reaching insertion of seta S4 . . . . . . A. armeniacus Arutunjan and Ohandjanian, 1972 —Seta Z4 short, less than 1/3 of distance between setae Z4 and S4 . . . . . A. kadzhajai Gomelauri, 1968

\section{Amblyseius andersoni (Chant, 1957)}

Material examined (open field): Perşembe, 23.07.2013, C. sativus (2우), 27.09.2013, C. annuum $(3 ㅇ ㅜ, 1$ ㄱ).

Material examined (greenhouse): Fatsa, 13.09.2013, S. melongena (1+). 
Distribution: $A$. andersoni is a very common predatory mite with a worldwide distribution (Demite et al., 2017). It has been reported together with various mite species by many researchers in Turkey (Faraji et al., 2011). In this study, it was collected with $B$. lewisi, $T$. urticae and $H$. staerki from $C$. annuum and $C$. sativus leaves in the open fields and with $T$. urticae and $H$. staerki on S. melongena in the greenhouses.

\section{Amblyseius rademacheri Dosse, 1958}

Senior synonym: Amblyseius khnzoriani Wainstein \& Arutunjan (Wainstein, 1975)

Material examined (open field): Ulubey, 11.09.2014, S. melongena (2q $q$ ).

Description $(n=2)$

Dorsum (Figure 2A) - Dorsal shield elongate, strongly reticulated; length (j1-J5) 343-345; width (s4s4) 165-167; seven pairs of solenostomes (gd1, gd2, gd4, gd5, gd6, gd8, gd9); 17 pairs of setae. Dorsal setae, short and minute regular, except for j1, j3, s4, Z4 and Z5. Z4 and Z5 strongly serrated. Peritremes extending beyond bases of setae $\mathrm{j} 1$ (Figure $2 \mathrm{~A}$ ).

Measurements of dorsal setae - j1 22-24, j3 33-35, j4 5-6, j5 4, j6 8-9, J2 9, J5 8, z2 12-13, z4 14, z5 6, Z1 9, Z4 86-89, Z5 108-109, s4 65-68, S2 11, S4 9, S5 8-9, r3 12-14, R1 8-9.

Venter (Figure 2B) - Sternal shield reticulated; length (ST1-ST3) 65-67; width (ST2-ST2) 66-68; two pairs of solenostomes (pst1-pst2); threepairs of setae (ST1, ST2 and ST3). Metasternal shield is located separately and bearing ST4. Genital shield length 76 (ST5-ST5). Ventrianal shield slightly reticulated; length 108-109, width (ZV2-ZV2) 98-105; three pairs of preanal setae (JV1, JV2 and ZV2); one pairs of crescentic solenostomes (gv3) located between setae JV2. Additionally, setae ZV1, ZV3, JV4 and JV5 surrounding ventrianal shield. Setae JV5 smooth, 50 in length.

Spermatheca (Figure 3A) - Calyx cup shaped; atrium C-like.

Chelicerae (Figure 3B) - Fixed digit of chelicerae with seven teeth and movable digit with two teeth, both 30-31 long.

Legs (Figure 3C) - Length of legs as follows: legl 341-358, legll 275-296, legllI 299-303 and leg IV 405-411. Three macrosetae on Leg IV (SgelV 58-59, StilV 39-40, StIV 82). Leg I, II and III with one macrosetae on genu (Sgel 26-34, Sgell 30-32, Sgelll 34).

Male - Not found in this study.

Remarks: In previous studies, seven species belonging to the genus Amblyseius (Faraji et al., 2011; Döker et al., 2014a; Akyazı et al., 2016b) were recorded for the Turkish fauna. Here, Amblyseius rademacheri is reported for the first time in Turkey.

Based on the female specimens, A. rademacheri is similar Amblyseius bryophilus Karg. However, it differs from the latter in having seven setae on genu II. A. bryophilus has 8 setae on genu II.

Amblyseius rademacheri is also similar to $A$. swirskii. However, it differs from the latter by having dorsal shield strongly reticulated, setae Z4 and Z5 long and serrate and two teeth on movable digit of chelicerae. Dorsal shield of $A$. swirskii is smooth, setae Z4 and Z5 slender and lightly serrate and three teeth on movable digit of chelicerae. Additionally, setae $\mathrm{S} 2$ of $A$. rademacheri are $11 \mu \mathrm{m}$ long while in that of $A$. swirskii approximately twice as long.

Amblyseius rademacheri was found together with $T$. urticae on S. melongena in this study. In previous studies, it was found on various fruits, weeds, forest trees in association with tetranychid and eriophid mites (Hajizadeh, 2007). Tixier et al. (2013) also reported A. rademacheri on Vitis vinifera L. (Vitaceae). Komi et al. (2008), found this species on pepper and eggplant in Japan.

Distribution: A. rademacheri has been recorded in Armenia, Austria, Azerbaijan, China-Jiangxi, Czech Republic, Denmark, Georgia, Germany, Hungary, Iran, Italy, Japan, Latvia, Moldova, Netherlands, Poland, Russia-Moscow Province, Primorsky Territory; Yaroslavl Province; Slovakia, Slovenia, South Korea, Spain, Switzerland, Ukraine (Demite et al., 2017) and Turkey (this study). 

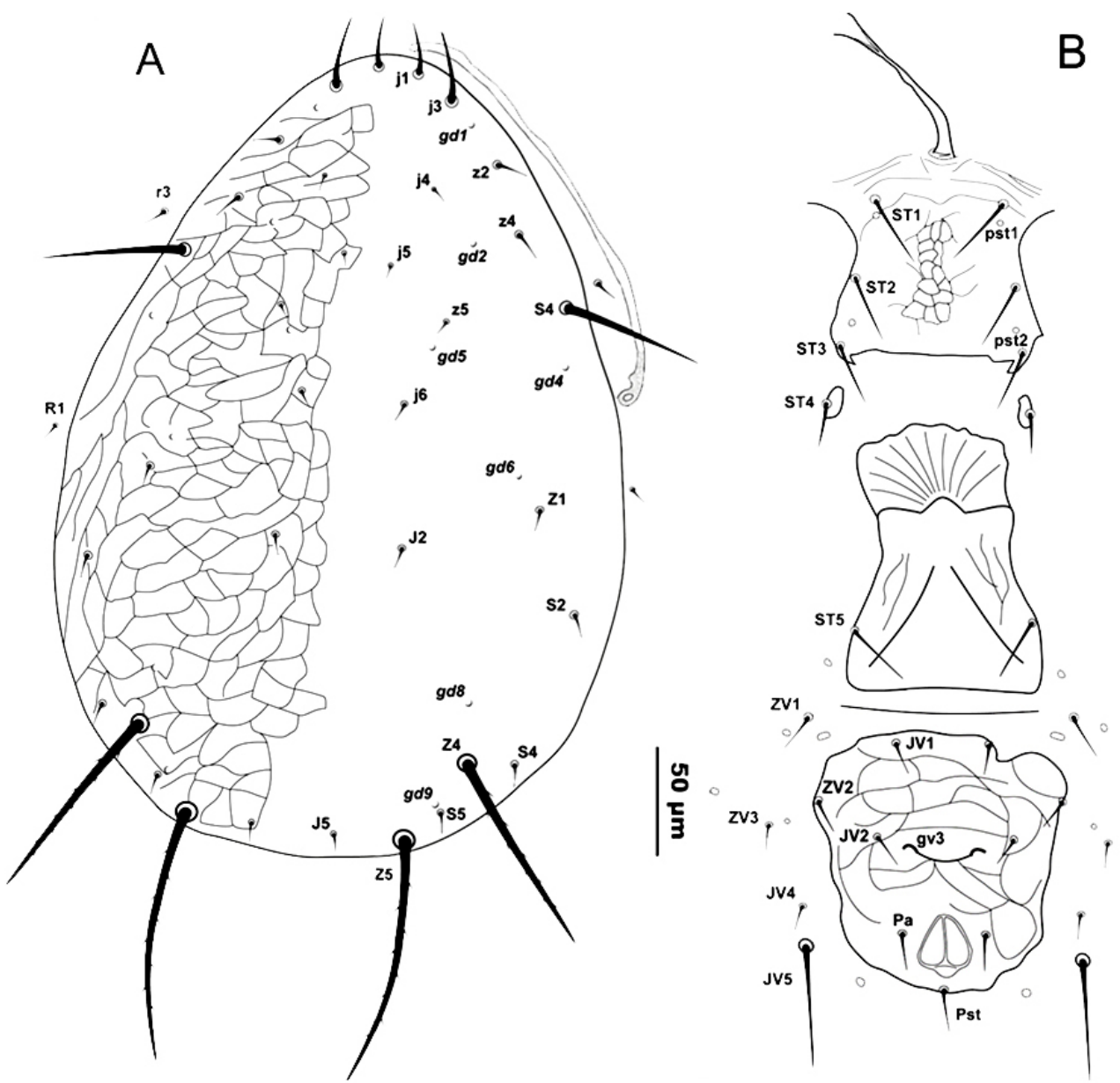

Figure 2. Dorsum (A) and venter (B) of Amblyseius rademacheri ( + ).

A

B
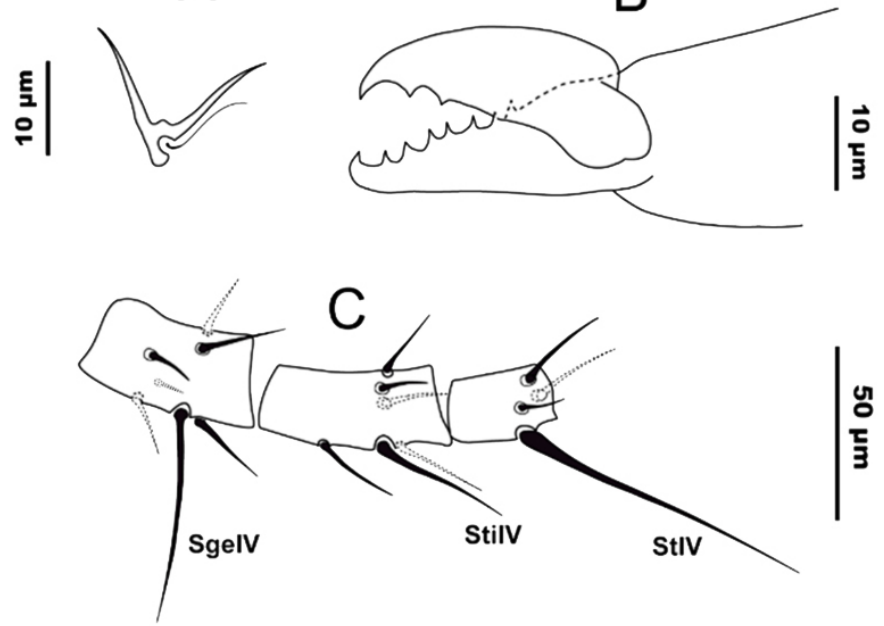

Figure 3. Spermatheca (A), chelicerae (B), and leg IV (C) of Amblyseius rademacheri ( + ). 


\section{Amblyseius swirskii Athias-Henriot, 1962}

Material examined (open field): Çaybaşı, 10.10.2014, P. vulgaris (2oq), C. sativus (13q $q$ ), S.

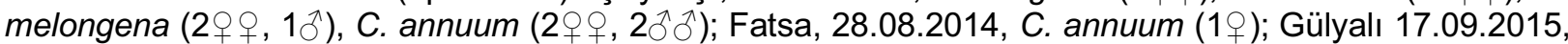
C. sativus (3우), S. melongena (1ㅇ); Altınordu, 21.07.2015, P. vulgaris (3우), C. sativus (9우); Perşembe, 27.09.2013, C. annuum (1dn), 09.07.2014, C. annuum (19); Ulubey, 06.09.2013, $P$. vulgaris

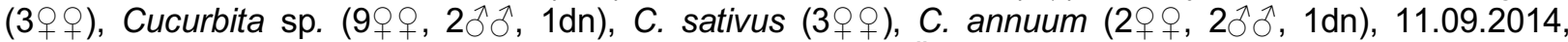

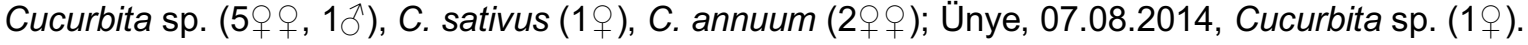

Distribution: $A$. swirskii is an important biological control agent of mites and small insects (Demite et al., 2017). This predator was reported for the first time on $P$. vulgaris, S. melongena and $C$. sativus in Adana (Kibritçi et al., 2007). In present study, it was collected in association with colonies of $B$. lewisi on C. annuum and Cucurbita sp., B. obovatus and T. palmarum on C. sativus and with $T$. urticae on all examined vegetable species.

\section{Aristadromips masseei (Nesbitt, 1951)}

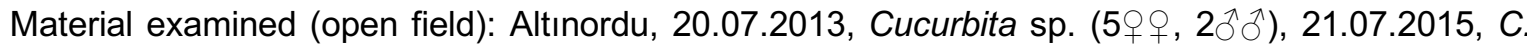
sativus (1+); Gülyalı, 10.07.2013, C. sativus (1+).

Material examined (greenhouse): Gürgentepe, 02.10.2014, C. sativus $(2 \circ++, 4 \hat{\jmath})$.

Distribution: $A$. masseei was determined in northwestern countries of the Palearctic region (Demite et al., 2017). This predatory was found for the first time in Giresun Province, Turkey (Çobanoğlu, 19911992). In this study, it was collected together with $T$. urticae from C. sativus and Cucurbita sp. leaves.

\section{Euseius finlandicus (Oudemans, 1915)}

Material examined (open field): Çaybaşı, 10.10.2014, C. annuum (2o 9 ); Çatalpınar, 21.08.2014, P. vulgaris (1); Kabadüz, 26.09.2014, C. annuum (3우); Perşembe, 27.09.2013, C. sativus (1ㅇ).

Distribution: E. finlandicus is common around the world (Demite et al., 2017). In Turkey, this predatory mite was also determined by many researchers (Faraji et al., 2011). During this research, $E$. finlandicus was collected with $T$. urticae on $P$. vulgaris and $C$. sativus.

\section{Euseius gallicus Kreiter and Tixier, 2010}

Material examined (open field): Gülyalı, 19.08.2015, $P$. vulgaris (7우), C. sativus $(10 \circ+$, $2 \hat{\delta} \widehat{\jmath}), C$. annuum (1).

Distribution: E. gallicus has been reported in Tunisia (Kreiter et al., 2010), Belgium, France, Germany, the Netherlands and Turkey (Tixier et al., 2009; Döker et al., 2014b). In this research, it was found together with Xenotarsonemus sp., T. goetzi and B. mali on C. annuum.

\section{Kampimodromus aberrans (Oudemans, 1930)}

Material examined (open field): Kabadüz, 26.09.2014, C. sativus (19); Perşembe, 09.07.2014, Cucurbita sp. (1§).

Distribution: Cosmopolitan K. aberrans has been recorded worldwide (Demite et al., 2017). It is one of the most common species in Turkey (Faraji et al., 2011). During this study, it was collected together with $T$. goetzi on Cucurbita sp. and T. urticae on C. sativus.

\section{Neoseiulus barkeri Hughes, 1948}

Material examined (open field): Fatsa, 28.08.2014, Cucurbita sp. (2 9 ); Perşembe, 27.09.2013, $P$. vulgaris (1), S. lycopersicum (1), S. melongena (1); Ulubey, 11.09.2014, S. melongena (2우).

Material examined (greenhouse): Perşembe, 23.07.2013, C. sativus (1+1).

Distribution: N. barkeri has been found in many countries around the world (Demite et al., 2017). In Turkey, it was determined on S. melongena in Antalya (Çobanoğlu, 1989a) and A. cepa in İzmir (Kılıç et al., 2012). It was found in association with $T$. urticae, B. obovatus on S. melongena and $T$. urticae on Cucurbita sp. in this study. 


\section{Neoseiulus bicaudus (Wainstein, 1962)}

Material examined (open field): Ünye, 07.08.2014, A. cepa (1+ ).

Distribution: N. bicaudus has been reported in Palearctic region (Asali Fayaz \& Khanjani, 2012). In Turkey, it was found on C. sativus (İnal, 2005) and S. melongena, Cucurbita sp. (Can \& Çobanoğlu, 2010) with T. cinnabarinus. In this study, N. bicaudus was collected together with population of $T$. urticae on $A$. cepa.

\section{Neoseiulus californicus (McGregor, 1954)}

Material examined (open field): Fatsa, 13.09.2013, S. melongena (19); Perşembe, 09.07.2014, Z. mays (1ㅇ).

Material examined (greenhouse): Perşembe, 23.07.2013, C. annuum (1ㅇ); Ünye, 07.08.2014, C. sativus $(13$ 우, 1 ㄱ).

Distribution: $N$. californicus is widely used as an effective biological control agent around the world. It has been found in many countries of the world (Demite et al., 2017). In Turkey, it was reported on $C$. annuum and $P$. vulgaris in association with $T$. urticae in Aydın (Çakmak \& Çobanoğlu, 2006). Çobanoğlu \& Kumral (2014) found $N$. californicus in association with populations of tetranychids in tomato fields in Ankara and Bursa. Döker et al. (2016) also determined this predatory mite with T. urticae on $S$. melongena.

\section{Phytoseius finitimus Ribaga, 1904}

Material examined (open field): Çatalpınar, 21.08.2014, S. melongena (1q); Gülyalı, 19.08.2015, P. vulgaris (1ㅇ), S. lycopersicum (2우); Kabadüz, 26.09.2014, P. vulgaris (2우); Altınordu, 23.09.2013,

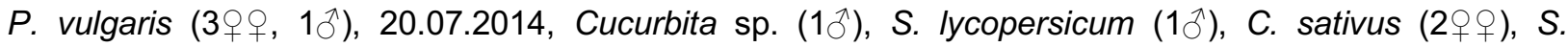

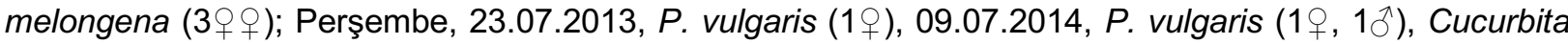
sp. (1pn), C. sativus (1)); Ulubey, 06.09.2013, P. vulgaris (1ㅇ), Cucurbita sp. (1ㅇ), 11.09.2014, C. annuum (19); Ünye, 26.07.2013, C. sativus (1), 07.08.2014, C. annuum (1+).

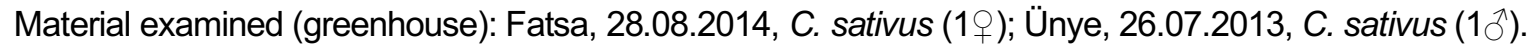

Distribution: $P$. finitimus is a common predatory mite species (Demite et al., 2017). It has been collected together with several mite species in many provinces of Turkey by many researchers (Faraji et al., 2011). In the current study, it was collected with T. urticae, A. pulvinum, P. latus, T. goetzi, T. putrescentiae, B. obovatus, T. immanis and $H$. staerki on $C$. annuum, P. vulgaris, S. melongena, $S$. lycopersicum and C. sativus.

\section{Phytoseiulus persimilis Athias-Henriot, 1957}

Material examined (open field): Çaybaşı, 10.10.2014, P. vulgaris (5우, 1pn), C. sativus (1ㅇ); Çatalpınar, 21.08.2014, P. vulgaris (10우, 1§), S. melongena (1ㅇ); Fatsa, 13.09.2013, S. lycopersicum $(1 \hat{)}), 28.08 .2014$, P. vulgaris $(1$,, $4 \hat{\jmath}$, 1pn); Gülyalı, 19.08.2015, P. vulgaris (1ㅇ), 17.09.2015, P. vulgaris (7우, 1 $\left.\delta^{\Uparrow}\right)$; Altınordu, 21.07.2015, C. sativus (1ㅇ); Perşembe, 29.07.2014, Cucurbita sp. (2우우); Ünye, 07.08.2014, P. vulgaris (1ㅇ, 2pn, 4tn), C. lanatus (1ㅇ).

Material examined (greenhouse): Ünye, 26.07.2013, C. sativus (15우, 1§), C. annuum (1ㅇ).

Distribution: P. persimilis is a common predatory species around the world (Demite et al., 2017) and has been successfully used for years for the biological control of $T$. urticae in many countries. Natural populations of $P$. persimilis have been recorded in Turkey (Şekeroğlu \& Kazak 1993; İnal, 2005; Akyazı \& Ecevit, 2008; Kasap et al., 2013; Çobanoğlu \& Kumral, 2014). 


\section{Proprioseiopsis okanagensis (Chant, 1957)}

Material examined (open field): Kabadüz, 26.09.2014, Cucurbita sp. (19).

Distribution: P. okanagensis was reported in Europe, North America (Demite et al., 2017) and Turkey (Çobanoğlu, 1989c; Çobaoğlu \& Bayram, 1999).

\section{Transeius wainsteini (Gomelauri, 1968)}

Material examined (open field): Çaybaşı, 10.10.2014, C. annuum (19); Gürgentepe, 02.10.2014, Cucurbita sp. (19); Perşembe, 23.07.2013, C. sativus (19), 27.09.2013, C. annuum (1ㅇ); Ünye, 07.08.2014, Cucurbita sp. (19).

Distribution: Demite et al. (2017) listed T. wainsteini in Armenia, Azerbaijan, Denmark, Georgia, Germany, Russia, Iran and Poland and Turkey. In this study, it was collected together with $B$. lewisi, $H$. staerki and T. urticae on C. annuum, Cucurbita sp. and C. sativus.

\section{Typhlodromus athiasae Porath and Swirski, 1965}

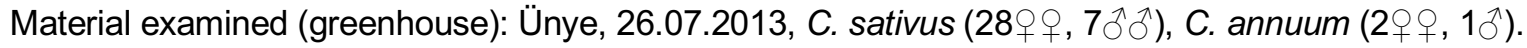

Distribution: T. athiasae is widespread around the world (Azerbaijan, Cyprus, Egypt, France, Greece, Iran, Israel, Jordan and Syria) (Demite et al., 2017). In Turkey, this predatory mite has been found by many researchers (Faraji et al., 2011).

\section{Conclusion}

A total of 43 mite species were identified during this study with $27 \%$ these found in both open fields and greenhouses. The others were found only in the open fields. Our results showed that the vegetables growing areas of Ordu Province, especially in open field conditions, are extremely rich with regard to beneficial mite fauna. This may be due to the limited usage of pesticides in the area. Therefore, the results obtained from the study may help to evaluate potential of the predators for biological control of phytophagous mites, including T. urticae and P. latus in Ordu. Amblyseius rademacheri, a promising predator was also found for the first time in Turkey. Therefore, in further studies it should be determined if A. rademacheri can be used in biological control programs in Turkey.

\section{Acknowledgments}

This research was supported by the Ordu University Scientific Research Project Coordination Unit (ODUBAP; Project No, TF-1304). The authors are grateful to Prof. Dr. Sultan Çobanoğlu (Phytoseiidae), Prof. Dr. Eddie A. Ueckermann (Cheyletidae, Eriophyidae, Erythraeidae, Tarsonemidae, Tetranychidae, Phytoseiidae, Prof. Dr. Salih Doğan (Stigmaeidae), Prof. Dr. Andrzej Kazmierski (Iolinidae, Tydeidae, Triophtydeidae), Prof. Dr. Alireza Saboori (Trombidiidae, Eupodidae, Erythraeidae), Prof. Dr. Antonio C. Lofego (Tarsonemidae) and Dr. Tea Arabuli (Tenuipalpidae) for the confirmation of species identification. We are also very thankful to anonymous reviewers for their deep, thorough review and constructive comments that helped us to improve this report.

\section{References}

Abak, K., F. Düzyaman, V. Şeniz, H. Gülen, A. Pekşen \& H. Ç. Kaymak, 2010. "Sebze üretimini geliştirme yöntem ve hedefleri, 477-492". Ziraat Mühendisliği VII. Teknik Kongresi (11-15 Ocak 2010, Ankara) Bildirileri, $1300 \mathrm{s.}$

Akyazı, F. \& O. Ecevit, 2003. Determination of mite species in hazelnut orchards in Samsun, Ordu and Giresun Provinces. Ondokuz Mayıs Üniversitesi Ziraat Fakültesi Dergisi, 18 (3): 39-45.

Akyazı, R. \& O. Ecevit, 2008. Samsun ili hıyar seralarında predatör akar Phytoseiulus persimilis Athias-Henriot (Acarina: Phytoseiidae)'in dağılımı. Mustafa Kemal Üniversitesi Ziraat Fakültesi Dergisi, 13 (1-2): 73-85.

Akyazı, R., E. A. Ueckermann, M. Soysal \& D. Akyol, 2016a. Population dynamics of mites (Acari) on Diospyros kaki Thunb. and Diospyros lotus L. (Ebenaceae) trees in Ordu, Turkey. Systematic and Applied Acarology, 21 (10): 1334-1345. 
Akyazı, R., E. A. Ueckermann, M. Soysal \& D. Akyol, 2016b. The new distribution of Amblyseius herbicolus in Turkey (Parasitiformes, Phytoseiidae) with a key of Amblyseius species found in Turkey. Acarologia, 56 (2): $237-244$.

Akyazı, R., E. A. Ueckermann, D. Akyol \& M. Soysal, 2017. Distribution of mite species (Acari) on persimmon trees in Turkey (Ordu), with one newly recorded mite species and one re-described species. International Journal of Acarology, 43 (8): 563-581.

Al-Atawi, F. J., 2011. Phytophagous and predaceous mites associated with vegetable crops from Riyadh, Saudi Arabia. Saudi Journal of Biological Sciences, 18 (3): 239-46.

Amrine, J. W. \& D. C. M. Jr Manson, 1996. "Preparation, mounting and descriptive study of eriophyoid mites, 383396". In: Eriophyoid Mites: Their Biology, Natural Enemies and Control (Eds. E. E. Lindquist, M. W. Sabelis \& J. Bruin). World Crop Pests, Volume 6. Elsevier, Amsterdam, 785 pp.

Andre, H. M., 2011. Dugés' caudatus is a Tenuipalpidae and not a Tydeidae (Acari). Acarologia. 51 (1): 69-85.

Anonymous, 2008. Zirai Mücadele Teknik Talimatları. T.C. Tarım ve Köyişleri Bakanlığı Koruma ve Kontrol Genel Müdürlüğü, Yenimahalle, Ankara, $332 \mathrm{~s}$.

Anonymous, 2015. Bibliography for "Lorryia mali" Biodiversity Heritage Library. (Web page: www.biodiversitylibrary.org/name/Lorryia_mali) (Date accessed: November 2015).

Asali Fayaz, B. \& M. Khanjani 2012. Phytoseiid mites (Acari: Mesostigmata: Phytoseiidae) in some regions of western and north western Iran. Journal of Crop Protection, 1 (2): 161-172.

Atyeo, W. T., 1960. A revision of the mite family Bdellidae in North and Central America (Acarina, Prostigmata). University of Kansas Science Bulletin, 40: 345-499.

Auger, P., A. Migeon, E. A. Ueckermann, L. Tiedt \& M. Navajas, 2013. Evidence for synonymy between Tetranychus urticae and Tetranychus cinnabarinus (Acari Prostimata, Tetranychiae): Review and new data. Acarologia, 53 (4): 383-415.

Baker, E. W. \& G. W. Wharton, 1952. An Introduction to Acarology. Macmillan, New York, 465 pp.

Beard, J. J., R. Ochoa, G. R. Bauchan, M. D. Trice, A. J. Redford, T. W. Walters \& C. Mitter, 2012. Flat Mites of the World Edition 2. Identification Technology Program, CPHST, PPQ, APHIS, USDA; Fort Collins, CO. (Web page: idtools.org/id/mites/flatmites) (Date accessed: November 2018).

Binisha, K. V. \& H. Bhaskar, 2013. Mite fauna associated with major vegetable crops of thrissur district, Kerala. Entomon, 38 (1): 47-52.

Bulut, E., 1999. Antalya ve Çevresinde Sebze Seralarında Bulunan Zararlılar ve Doğal Düşmanların Saptanması. Akdeniz Üniversitesi Fen Bilimleri Enstitüsü Bitki Koruma Anabilim Dalı, (Basılmamış) Yüksek Lisans Tezi, Antalya, $70 \mathrm{~s}$.

CABI, 2014. Polyphagotarsonemus latus (Broad Mite). (Web page: www.cabi.org/isc/datasheet/26876) (Date accessed: November 2015).

Çakmak, I. \& S. Çobanoğlu, 2006. Amblyseius californicus (McGregor, 1954) (Acari: Phytoseiidae), a new record for the Turkish Fauna. Turkish Journal of Zoology, 30 (1): 55-58.

Can, M. \& S. Çobanoğlu, 2010. Kumluca (Antalya) ilçesinde sebze üretimi yapılan seralarda bulunan akar (Acari) türlerinin tanımı ve konukçuları üzerinde çalışmalar. Akdeniz Üniversitesi Ziraat Fakültesi Dergisi, 23 (2): $87-92$.

Çelik, N., 2009. Samsun İlinde Ev Tozu Akarı Türlerinin Belirlenmesi ve Alerjik Astım İle Arasındaki İlişkinin Ortaya Konulması. Ondokuzmayıs Üniversitesi, Fen Bilimleri Enstitüsü, (Basılmamış) Yüksek Lisans Tezi, Samsun, 84 s.

Çıkman, E., A. Yücel \& S. Çobanoğlu, 1996. "Şanlıurfa ili sebze alanlarında bulunan akar türleri, yayılışları ve konukçuları, 517-525”. Türkiye 3. Bitki Koruma Kongresi (24-28 Eylül, 1996, Ankara) Bildirileri, 380 s.

Çobanoğlu, S., 1989a. Antalya ili sebze alanlarında tespit edilen Phytoseiidae Berlese, 1915 (Acarina: Mesostigmata) türleri. Bitki Koruma Bülteni, 29 (1-2): 47-64.

Çobanoğlu, S., 1989b. Türkiye'nin bazı turunçgil bölgelerinde tespit edilen faydalı akar (Acarina: Phytoseiidae) türleri. Türkiye Entomoloji Dergisi, 13 (3): 163-178.

Çobanoğlu, S., 1989c. Türkiye için üç yeni faydalı akar (Acari: Phytoseiidae) türü. Türkiye Entomoloji Dergisi, 13 (4): 229-38. 
Mite species of the vegetable crops in Ordu Province with first report of Amblyseius rademacheri Dosse, 1958 (Mesostigmata: Phytoseiidae) in Turkey

Çobanoğlu, S., 1991-1992. An annoted list of mites on hazel of Turkey. Israel Journal of Entomology, 25: 35-40.

Çobanoğlu, S. 1993a. Türkiye'nin önemli elma bölgelerinde bulunan Phytoseiidae (Parasitiformes) türleri üzerinde sistematik çalışmalar I. Türkiye Entomoloji Dergisi, 17 (1): 41-54.

Çobanoğlu, S. 1993b. Türkiye'nin önemli elma bölgelerinde bulunan Phytoseiidae (Parasitiformes) türleri üzerinde sistematik çalışmalar II. Türkiye Entomoloji Dergisi, 17 (2): 99-116.

Çobanoğlu, S. 1993c. Türkiye'nin önemli elma bölgelerinde bulunan Phytoseiidae (Parasitiformis) türleri üzerinde sistematik çalışmalar III. Türkiye Entomoloji Dergisi, 17 (3): 175-192.

Çobanoğlu, S. 1993d. Türkiye'nin önemli elma bölgelerinde bulunan Phytoseiidae (Parasitiformis) türleri üzerinde sistematik çalışmalar IV. Türkiye Entomoloji Dergisi, 17 (4): 239-255.

Çobanoğlu, S., 1995. Some new tarsonemidae (Acarina, Prostigmata) species for Turkish acarofauna. Türkiye Entomoloji Dergisi, 19 (2): 87-94.

Çobanoğlu, S. \& A. Kazmierski, 1999. Tydeidae and Stigmeidae (Acari, Prostigmata) from orchards, trees and shurbs in Turkey. Biological Bulletin of Poznań, 36: 71-82.

Çobanoğlu, S. \& S. Bayram, 1999. Mite (Acari) species associated with cultivated and wild rose plants in Camlidere, Turkey. Entomologist's Monthly Magazine, 135: 245-248.

Çobanoğlu, S., C. Uysal \& E. Ökten, 2003. The complex of the beneficial mite fauna of ornamental trees and shrubs in Ankara, Turkey. Entomologist's Monthly Magazine, 139: 7-12.

Çobanoğlu, S. \& N. A. Kumral, 2014. Ankara, Bursa ve Yalova illerinde domates yetiştirilen alanlarda zararlı ve faydalı akar (Acari) biyolojik çeşitliliği ve popülasyon dalgalanması. Türkiye Entomoloji Dergisi, 38 (2): 197-214.

Çobanoğlu, S. \& N. A. Kumral, 2016. The biodiversity, density and population trend of mites (Acari) on Capsicum annuum L. in temperate and semi-arid zones of Turkey. Systematic and Applied Acarology, 21 (7): 907-918.

Çobanoğlu, S., E. A. Ueckermann \& H. D. Sağlam, 2016. The Tenuipalpidae of Turkey, with a key to species (Acari: Trombidiformes). Zootaxa, 4097 (2): 151-186.

Demite, P. R., G. J. de Moraes, J. A. McMurtry, H. A. Denmark \& R. C. Castilho, 2017. Phytoseiidae Database. (Web page: www.lea.esalq.usp.br/phytoseiidae) (Date accessed: November 2017).

Denizhan, E. \& S. Çobanoğlu, 2009. Ankara ili ceviz (Juglans regia L.) ağaçlarında bulunan eriophyid akarlar ve predatörleri. Yüzüncü Yıl Üniversitesi Tarım Bilimleri Dergisi, 19 (1): 33-37.

Denizhan, E., R. Monfreda, E. De Lillo \& S. Çobanoğlu, 2015. Eriophyoid mite fauna (Acari: Trombidiformes: Eriophyoidea) of Turkey: New species, new distribution reports and an updated catalogue. Zootaxa, 3991 (1): 1-63.

Döker, I., Thl. Stathakis \& C. Kazak, 2014a. First record of Amblyseius bryophilus Karg (Acari: Phytoseiidae) for the Turkish fauna. Turkish Journal of Zoology, 38 (3): 375-377.

Döker, I., J. Witters, J. Pijnakker, C. Kazak, M. S. Tixier \& S. Kreiter, 2014b. Euseius gallicus Kreiter and Tixier (Acari: Phytoseiidae) is present in four more countries in Europe: Belgium, Germany, The Netherlands and Turkey. Acarologia, 54 (3): 245-248.

Döker, I., C. Kazak \& K. Karut, 2016. Functional response and fecundity of a native Neoseiulus californicus population to Tetranychus urticae (Acari: Phytoseiidae, Tetranychidae) at extreme humidity conditions. Systematic and Applied Acarology, 21 (10): 1463-1472.

Dönel, G. \& S. Doğan, 2013. Predatör bir akar olan Zetzellia mali (Ewing) (Acari: Stigmaeidae)'nin Kelkit Vadisi'nden ilk kaydı. Erzincan Üniversitesi Fen Bilimleri Enstitüsü Dergisi, 6 (2): 157-163.

Düzgüneş, Z., 1952. Türkiye'de turunçgil akarları. Bitki Koruma Bülteni, 1: 6-11.

Düzgüneş, Z., 1963. Türkiyede yeni bulunan akarlar. Bitki Koruma Bülteni, 3 (4): 237-46.

Ecevit, O., 1976. Akar (Acarina)'ların Toplanması, Saklanması ve Preparatlarının Yapılması. Atatürk Üniversitesi Yayınları, Ankara, Yayın No: 480, 32s.

Edward, W. \& M. Donald, 1987. The False Spider Mites of Mexico (Tenuipalpidae: Acari). Agricultural Research Service, Tectinical Bulletin, $241 \mathrm{pp}$.

Ewing, H. E., 1917. New Acarina. Part II. Description of new species and varieties from lowa, Missouri, Illinois, Indiana and Ohio. American Museum of Natural History, 37 (2): 149-172. 
Fain, A, R. L. Smiley \& U. Gerson, 1999. Further observations on the Cheyletidae (Acari), with a key to the genera of the Cheyletinae and a list of all known species in the family. Bulletin de \{l'Institute\} Royal des Sciences Naturelles de Belgique, Entomologie, 69 (1977): 35-86.

Fan, Q. H. \& Z. Q. Zhang, 2005. Raphignathoidea (Acari: Prostigmata). Fauna of New Zealand, 52 (1): 1-400.

Fan, Q. H. \& Z. Q. Zhang, 2007. Tyrophagus (Acari: Astigmata: Acaridae). Fauna of New Zealand, 56: 1-291.

Faraji, F., J. Hajizadeh, E. A. Ueckermann, K. Kamali \& J. A. McMurtry, 2007. Two new records for Iranian phytoseiid mites with synonymy and keys to the species of Typhloseiulus Chant \& MacMurtry and Phytoseiidae in Iran (Acari: Mesostigmata). International Journal of Acarology, 33 (3): 231-239.

Faraji, F., S. Çobanoğlu \& I. Çakmak, 2011. A checklist and a key for the Phytoseiidae species of Turkey with two new species records (Acari: Mesostigmata). International Journal of Acarology, 37 (suppl.1): 221-243.

Franz, J. T., G. Masuch, H. Musken \& K. C. Bergmann, 1997. Mite fauna of German farms. Allergy, 52 (12): $1233-1237$.

Gerson, U., R. L. Smiley \& R. Ochoa, 2003. Mites (Acari) for Pest Control. Blackwell Science, 558 pp.

Ghai, S. \& M. Shenhmar, 1984. A review of the world fauna of Tenuipalpidae (Acarina: Tetranychoidea). Oriental Insects, 18: 99-172.

Gonzalez-Rodrigeuz, R. H., 1965. A taxonomic study of the genera Mediolata, Zetzellia and Agistemus (Acarina: Stigmaeidae). University of California Publications in Entomology, 41: 1-64.

Göven, M. A., S. Çobanoğlu \& B. Güven 2009. Ege Bölgesi bağ alanlarındaki avcı akar faunası. Bitki Koruma Bülteni, 49 (1): 1-10.

Gupta, S. K., 1985. Plant Mites of India. Zoological Survey of India Calcutta, 564 pp.

Gupta, S. K., J. Mondal \& B. Chakraborty, 2015. Diversity of some mites occurring on medicinal plants in South Bengal with New records of hosts/habitats along with their economic Importance. Global Journal for Research Analysis, 4 (10): 257-60.

Güven, B. \& N. Madanlar, 2000. Tertanychus urticae Koch (Acarina: Tetranychidae)'nin Salihli (Manisa)'de ikinci ürün mısırda populasyon yoğunluğu ve laboratuvarda iki farklı mısır çeşidinde bazı biyolojik özellikleri üzerinde araştırmalar. Türkiye Entomoloji Dergisi, 24 (4): 279-288.

Hajizadeh, J., 2007. Fauna of Phytoseiidae mites (Acari: Phytoseiidae) in Guilan Province. Final report of research project. Guilan, Iran: Agricultural Sciences College, University of Guilan, 64 pp.

Hıncal, P., N. Yaşarakıncı \& I. Çınarlı, 2002. İzmir ilinde domates pas akarı (Aculops lycopersici Massee) (Acarina: Eriophyidae)'nın popülasyon seyri, doğal düşmanları ve kimyasal mücadelesi üzerinde araştırmalar. Bitki Koruma Bülteni, 42 (1-4): 9-22.

İnak, E. \& S. Çobanoğlu, 2018. Determination of mite species on vineyards of Ankara, Turkey. Fresenius Environmental Bulletin, 27 (2): 1232-1239.

İnal, B., 2005. Bafra ve Çarşamba Ovalarında Çeşitli Kültür Bitkisi Alanlarında Bulunan Acarina Türleri Üzerinde Faunistik Çalışmalar. Ondokuz Mayıs Üniversitesi Fen Bilimleri Enstitüsü Bitki Koruma Anabilim Dalı, (Basılmamış) Yüksek Lisans Tezi, Samsun, $100 \mathrm{~s}$.

Jalilirad, M., J. Hajicadeh \& J. Noei, 2012. Fauna of prostigmatic mites (Acari: Prostigmata) associated with citrus orchards in Guilan Province. Journal of Plant Pests Research, 2 (4): 1-13.

Jeppson, L. R., H. B. Keifer \& E. W. Baker, 1975. Mites Injurious to Economic Plants. University of California Press, 614 pp.

Kade, N., A. Gueye-Ndiaye, C. Duverney \& G. J. de Moraes, 2011. Phytoseiid mites (Acari: Phytoseiidae) from Senegal. Acarologia, 51 (1): 133-138.

Karagöz, B., 2010. Çanakkale İli Domates Alanlarında Zararlı Kırmızıörümcek Tetranchus cinnabarinus Biosduval (Acarina: Tetranychidae)'un Populasyon Gelişmesi ve Predatörlerin Belirlenmesi. Çanakkale Onsekiz Mart Üniversitesi Fen Bilimleri Enstitüsü Bitki Koruma Anabilim Dalı, (Basılmamış) Yüksek Lisans Tezi, Çanakkale, 38 s.

Kasap, İ. \& S. Çobanoğlu, 2007. Mite (Acari) fauna in apple orchards of around the Lake Van basin of Turkey. Türkiye Entomoloji Dergisi, 31 (2): 97-109.

Kasap, İ., S. Çobanoğlu \& S. Pehlivan, 2013. Çanakkale ve Balıkesir illeri yumuşak çekirdekli meyve ağaçları ve yabancı otlar üzerinde bulunan predatör akar türleri. Türkiye Biyolojik Mücadele Dergisi, 4 (2): 109-124. 
Mite species of the vegetable crops in Ordu Province with first report of Amblyseius rademacheri Dosse, 1958 (Mesostigmata: Phytoseiidae) in Turkey

Khanjani, M., S. Farzan, M. Asadi \& M. Khanjani, 2013. Checklist of the flat mites (Acari: Trombidiformes: Tenuipalpidae) of Iran. Persian Journal of Acarology, 2 (2): 235-251.

Kibritçi, C., C. Kazak \& K. Karut, 2007. Avcı akar Typhlodromips (Amblyseius) enab El-Badry (Acari: Phytoseiidae)'ın farklı sıcaklıklarda biyolojilerinin belirlenmesi. Türkiye Entomoloji Dergisi, 31 (2): 129-138.

Kılıç, T., S. Çobanoğlu, Z. Yoldaş \& N. Madanlar, 2012. İzmir ilinde taze soğan tarlalarında bulunan akar (Acari) türleri. Türkiye Entomoloji Dergisi, 36 (3): 401-411.

Komi, K., R. Arakawa \& H. Amano, 2008. Native phytoseiid mites (Acari: Phytoseiidae) occurring on greenhouse vegetable crops under the pest control programs with natural enemies in Kochi prefecture, Japan. Journal of the Acarological Society of Japan, 17 (1): 23-28.

Krantz, G. W. \& D. E. Walter, 2009. A Manual of Acarology. 3rd ed. Lubbock (TX), Texas Tech University Press, 816 pp.

Kreiter, S., M. S. Tixier, H. Sahraoui, K. Lebdi-Grissa, S. Ben Chabaan, A. Chatti, B. Chermiti, O. Khoualdia \& M. Ksantini, 2010. Phytoseiid mites (Acari: Mesostigmata) from Tunisia: Catalogue, biogeography, and key for identification. Tunisian Journal of Plant Protection, 5: 151-178.

Kumral, N. A., 2005. Bursa İlinde Ilıman İklim Meyvelerinde Bulunan Zararlı ve Doğal Düşman Akarların Saptanması ve Panonychus ulmi (Koch)'nin Bazı Pestisitlere Karşı Duyarlılığı Üzerine Araştırmalar. Uludağ Üniversitesi Fen Bilimleri Enstitüsü Bitki Koruma Anabilim Dalı, (Basılmamış) Doktora Tezi, Bursa, $172 \mathrm{~s}$.

Kumral, N. A. \& S. Çobanoğlu, 2015a. The potential of the nightshade plants (Solanaceae) as reservoir plants for pest and predatory mites. Türkiye Entomoloji Dergisi, 39 (1): 91-108.

Kumral, N. A. \& S. Çobanoğlu, 2015b. A reservoir weed for mites: Datura stramonium L. (Solanaceae) in the vicinity of cultivated solanaceous plants in Turkey. International Journal of Acarology. 41 (7): 563-573.

Lin, J. Z. \& Z. Q. Zhang, 2002. Tarsonemidae of the World: Key to Genera, Geographical Distribution, Systematic Catalogue \& Annotated Bibliography. Systematic \& Applied Acarology Society, London, 440 pp.

Madanlar, N., 1991. İzmir İlinde Turunçgillerde Bulunan Acarina Türleri ve Populasyon Yoğunluklarının Saptanması Üzerinde Araştırmalar. Ege Üniversitesi Fen Bilimleri Enstitüsü, Bitki Koruma Anabilim Dalı, (Basılmamış) Doktora Tezi, İzmir, $258 \mathrm{~s}$.

Madanlar, N. \& C. Öncüer, 1994. İzmir ilinde sera domatesiz olarak Aculops lycopersici (Massee) (Acarina, Eriophyidae). Türkiye Bitki Koruma Dergisi, 18 (4): 237-240.

Migeon, A., E. Nouguier \& F. Dorkeld, 2011. Spider Mites Web: A Comprehensive Database for the Tetranychidae. Trends in Acarology. (Web page: http://www.montpellier.inra.fr/CBGP/spmweb) (Date accessed: April 2016).

Muma, M. H. \& H. A. Denmark, 1970. "Phytoseiidae of Florida" arthropods of Florida and neigboring land areas. Florida Department of Agriculture, Division of Plant Industries, 6: 1-150.

Öngören, K., N. Kaya \& Ş. Türkmen, 1975. Ege bölgesi sebzelerinde zarar yapan kırmızı örümcek türlerinin tespiti, hakim tür olan Tetranychus urticae koch'nin biyolojisi, mücadelesi ve doğal düşmanları üzerinde araştırmalar. Bitki Koruma Bülteni, 15 (1): 3-29.

Özman-Sullivan, S. K., A. Kazmierski \& S. Çobanoğlu. 2005. Alycina and Eupodina mites of hazelnut orchards in Turkey. VI. Int. Hazelnut Congress, 14-18 June 2004, Tarragona, Spain, Acta Horticulturae, 686: 401-406.

Pritchard, A. E. \& E. W. Baker, 1955. A revision of the spider mites family Tetranychidae. Pacific Coast Entomological Society. 2: 472.

Radonjic, S. \& S. Hrncic, 2011. An overview of invasive species on vegetables in greenhouses in southern part of Montenegro. IOBC/WPRS Bulletin, 153-157 pp.

Ripka, G., A. Fain, A. Kazmierski, S. Kreiter \& W. Ł. Magowski, 2002. Recent data to the knowledge of the arboreal mite fauna in Hungary (Acari: Mesostigmata, Prostigmata, and Astigmata). Acarologia, 42 (3): 271-281.

Ripka, G., A. Fain, A. Kazmierski, S. Kreiter \& W. Ł. Magowski, 2005. New data to the knowledge of the mite fauna of Hungary (Acari Mesostigmata, Prostigmata and Astigmata). Acta Phytopathologica et Entomologica Hungarica, 40: 159-176.

Ripka, G., I. Laniecka \& A. Kazmierski, 2013. On the arboreal acarofauna of Hungary: Some new and rare species of prostigmatic mites (Acari: Prostigmata: Tydeidae, Iolinidae and Stigmaeidae). Zootaxa, 3702 (1): 1-50. 
Rowell, H. J., D. A. Chant \& R. I. C. Hansell, 1978. The determination of setal homologies and setal patterns on the dorsal shield in the family Phytoseiidae (Acarina: Mesostigmata). Canadian Journal of Zoology, 110: 859-876.

Sabbatini Peverieri, G., S. Simoni, D. Goggioli, M. Liguori \& M. Castagnoli, 2009. Effects of variety and management practices on mite species diversity in Italian vineyards. Bulletin of Insectology, 62 (1): 53-60.

Sağlam, H. D. \& S. Çobanoğlu, 2010. Determination of Tenuipalpidae (Acari: Prostigmata) species in parks and ornamental plants of Ankara, Turkey. Türkiye Entomoloji Dergisi, 34 (1): 37-52.

Schruft, G., 1972. Das Vorkommen von Milben aus der Familie Tydeidae (Acari) and Reben. VI. Beitrag über Untersuchungen zur Faunistik und Biologie der Milben an Kulturreben (Vitis sp.). Zeitschruft Für Angewandte Entomologie, 71: 124-133.

Schruft, G., 2006. The occurrence of mites of the family Tydeidae (Acari) on vines. VI. Contribution on investigations on the fauna and biology of mites (Acari) on cultivated vines (Vitis spp.). Zeitschrift fur Angewandte Entomologie (Web page: https://www.cabi.org/ISC/abstract/19740512761) (Date accessed: November 2009).

Seeman, O. D. \& J. J. Beard, 2011. Identification of exotic pest and Australian native and naturalised species of Tetranychus (Acari: Tetranychidae). Zootaxa, 72: 1-72.

Şekeroğlu, E., A. F. Özgür, 1984. A new tomato pest in Çukurova, Aculops lycopersici (Massee), (Acarina; Eriophyidae). Türkiye Bitki Koruma Dergisi, 8 (4): 211-213.

Şekeroğlu, E. \& C. Kazak, 1993. First record of Phytoseiulus persimilis (Acari: Phytoseiidae) in Turkey. Entomophaga, 38 (3): 343-345.

Skvarla, M., J. Fisher \& A. Dowling, 2014. A review of Cunaxidae (Acariformes, Trombidiformes): Histories and Diagnoses of subfamilies and genera, keys to world species, and some new locality records. ZooKeys, 418: 1-103.

Solarz, K. 2012. House Dust Mites and Storage Mites (Acari: Oribatida: Astigmatina) Identification Keys. Institute of Systematics and Evolution of Animals, Polish Academy of Sciences, Cracow, Poland, 120 pp.

Soylu, O. Z. \& N. Ürel 1977. Güney Anadolu Bölgesi turunçgillerinde zararlı böceklerin parazit ve predatörlerinin tesbitî üzerinde araştırmalar. Bitki Koruma Bülteni, 17 (2-4): 77-112.

Soysal, A. \& A. Yayla, 1988. Antalya ili patlıcanlarında zararlı Tetranychus spp. (Acarina: Tetranychidae)'nin ve doğal düşmanlarının populasyon yoğunlukları tespiti üzerine ön çalışmalar. Bitki Koruma Bülteni, 28 (1-2): 29-41.

Stojnic, B., H. Panou, G. Papadoulis, R. Petanovic \& N. Emmanouel, 2002. The present knowledge and new records of phytoseiid and tydeid mites (Acari: Phytoseiidae, Tydeidae) for the fauna of Serbia and Montenegro. Acta Entomologica Serbica, 7 (1/2): 111-117.

Summers, F. M. \& D. W. Price, 1970. Review of the mite family Cheyletidae. University of California Publications in Entomology, 61: 1-153.

Tempfli, B., B. Pénzes, J. Fail \& Á. Szabó, 2015. The occurrence of tydeoid mites (Acari: Tydeoidea) in Hungarian Vineyards. Systematic and Applied Acarology, 20 (8): 937-954.

Tixier, M. S., S. Kreiter, M. Okassa \& B. Cheval, 2009. A new species of the genus Euseius Wainstein (Acari: Phytoseiidae) from France, Journal of Natural History, 44 (3-4): 241-254.

Tixier, M. S., A. Baldassar, C. Duso \& S. Kreiter, 2013. Phytoseiidae in European grape (Vitis vinifera L.): Bioecological aspects and keys to species (Acari: Mesostigmata). Zootaxa, 3721 (2): 101-142.

Tokkamış, F. N. \& D. Yanar, 2011. "Tokat İlinde yetiştirilen bazı sebzelerde belirlenen zararlı ve faydalı akar türleri. 24". Türkiye 4. Bitki Koruma Kongresi (28-30 Haziran 2011, Kahramanmaraş) Bildirileri, 496 s.

Tokkamış, F. N., 2011. Tokat İlinde Yetiştirilen Bazı Sebze Türlerinde Faydalı ve Zararlı Akar (Acari) Türlerinin Belirlenmesi. Gaziosmanpaşa Üniversitesi, Fen Bilimleri Enstitüsü, Bitki Koruma Anabilim Dalı, (Basılmamış) Yüksek Lisans Tezi, Tokat, $69 \mathrm{~s}$.

Toros, S., 1974. Orta Anadolu Bölgesi'nde Önemli Bitki Zararlılarından Tetranychus viennensis Zacher (Akdiken akarı)'in Morfolojisi, Biyolojisi, Yayılışı ve Konukçuları ile Kimyasal Savaş İmkanları Üzerinde Araştırmalar. Ankara Üniversitesi Ziraat Fakültesi Yayınları, 514: $74 \mathrm{~s}$.

Tunç, I. \& H. Göçmen, 1995. Antalya'da bulunan iki sera zararlısı Polyphagotarsonemus latus (Banks) (Acarina, Tarsonemidae) ve Frankliniella occidentalis (Pergande) (Tysanoptera, Thripidae) üzerine notlar. Türkiye Entomoloji Dergisi, 19 (2): 101-109. 
Mite species of the vegetable crops in Ordu Province with first report of Amblyseius rademacheri Dosse, 1958 (Mesostigmata: Phytoseiidae) in Turkey

TÜIK, 2017. Bitkisel üretim istatistikleri. (Web page: www.tuik.gov.tr) (Date accessed: April 2018).

Ueckermann, E. A. \& T. G. Grout, 2007. Tydeoid mites (Acari: Tydeidae, Edbakerellidae, lolinidae) occurring on citrus in Southern Africa. Journal of Natural History, 41 (37-40): 2351-2378.

Ueckermann, E. A. \& S. Çobanoğlu, 2012. Phytophagous Mites of Economical Importance of Turkey. Workshop in Taxonomic Acarology, (21-22 June 2012, Ankara, Turkey), 61 pp.

Ueckermann, E. A., 2013a. Course on Taxonomy of the Tarsonemidae. 3. Workshop in Taxonomic Acarology, (0910 July 2013, Bursa, Turkey), 21 pp.

Ueckermann, E. A., 2013b. Course on Taksonomy of the Tydeoidae. 3. Workshop in Taxonomic Acarology, (09-10 July 2013, Bursa, Tukey), 24 pp.

Ueckermann, E. A. \& G. Ripka, 2016. Three new species and a new record of tenuipalpid mites (Acari: Tenuipalpidae) from Hungary. Journal of Natural History, 50 (15-16): 989-1015.

Ueckermann, E. A., E. Palevsky, U. Gerson, E. Recht \& P. D. Theron, 2018. The Tenuipalpidae (Acari: Trombidiformes) of Israel. Acarologia 58 (2): 483-525.

Volgin, V. I., 1989. Acarina of the Family Cheyletidae of the World. Amerind Publishing Co. Pvt. Ltd, New Delhi, 532 pp.

Yabaş, C. \& A. Ulubilir, 1995. Akdeniz Bölgesi'nde biberde yeni saptanan bir zararlı Polyphagotarsonemus latus (Banks) (Acarina, Tarsonemidae). Türkiye Entomoloji Dergisi, 19 (1): 43-46.

Yanar, D. \& H. Erdoğan, 2013. Tokat İlinde Bulunan Taş Çekirdekli Meyvelerde Bulunan Akar Türlerinin Belirlenmesi. Gaziosmanpaşa Üniversitesi Bitki Koruma Anabilim Dalı Bilimsel Araştırma Projeleri Bitirme Raporu, $100 \mathrm{~s}$.

Yanar, D., O. Ecevit \& I. Kadıoğlu, 2008. Tokat yöresinde domates ekim alanlarında zarar oluşturan domates pas akarı [Aculops lycopersici (Massee) (Acari: Eriophyidae)]. Gaziosmanpaşa Üniversitesi Ziraat Fakültesi Dergisi, 25 (2): 1-5.

Yeşilayer, A., 2009. İstanbul İli Yeşil Alanlarında Zararlı Akar (Acarina) Türlerinin Tanımı, Yayılışı, Önemli Türün Populasyon Yoğunluğu ve Doğal Düşmanları Üzerinde Araştırmalar. Ankara Üniversitesi Fen Bilimleri Enstitüsü Bitki Koruma Anabilim Dalı, (Basılmamış) Doktora Tezi, Ankara, $185 \mathrm{~s}$.

Yeşilayer, A. \& S. Çobanoğlu, 2012. Türkiye akar faunası ıçin yeni bir kayıt: Cheletomimus berlesei (Oudemans) (Acari: Cheyletidae). Türkiye Entomoloji Bülteni, 2 (3): 183-188.

Zdarkova, E., 1967. Stored food mites in Czechoslovakia. Journal of Stored Products Research, 3 (2): 155-175.

Zhang, Z. Q. \& H. Norbakhsh, 1995. A new genus and three new species of mites (Acari: Trombidiidae) described from larvae ectoparasitic on aphids from Iran. European Journal of Entomology, 92 (4): 705-718.

Zhang, Z. Q., 2000. Key to Tarsonemidae of New Zealand. Final report to MAF Science Policy for Project FMA102: 1-35.

Zhang, Z., R. Henderson, A. Flynn \& N. A. Martin, 2002. Key to Tetranychidae of New Zealand. Landcare Research Contract Report: LC0102/144, Prepared for: MAF Science Policy, Project FMA180, 62 pp.

Zhang, Z. Q., 2003. Mites of Greenhouses, Identification, Biology and Control, CABI Publishing, 256 pp. 